\title{
A Model for Tracing Variability from Features to Product-Line Architectures: A Case Study in Smart Grids
}

\author{
Jessica Díaz - Jennifer Pérez · Juan Garbajosa
}

\begin{abstract}
In current software systems with highly volatile requirements, traceability plays a key role to maintain the consistency between requirements and code. Traceability between artifacts involved in the development of Software Product Lines (SPL) is still more critical because it is necessary to guarantee that the selection of variants that realize the different SPL products meet the requirements. Current SPL traceability mechanisms trace from variability in features to variations in the configuration of product-line architecture (PLA) in terms of adding and removing components. However, it is not always possible to materialize the variable features of a SPL through adding or removing components, since sometimes they are materialized inside components, i.e. in part of their functionality: a class, a service and/or an interface. Additionally, variations that happen inside components may crosscut several components of architecture. These kinds of variations are still challenging and their traceability is not currently well-supported. Therefore, it is not possible to guarantee that those SPL products with these kinds of variations meet the requirements. This paper presents a solution for tracing variability from features to PLA
\end{abstract}

by taking these kinds of variations into account. This solution is based on models and traceability between models in order to automate SPL configuration by selecting the variants and realizing the product application. The FPLA modeling framework supports this solution which has been deployed in a software factory. Validation has consisted in putting the solution into practice to develop a product line of power metering management applications for Smart Grids.

Keywords Traceability Modeling - Software Product Line Engineering . Product-Line Architecture .

Variability

\section{Introduction}

Traceability defines and maintains relationships between artifacts involved in the software life cycle $[19,2]$ in both forward and backward directions, e.g. from requirements to code and from code to requirements, respectively. Currently, software systems are continuously undergoing changes due to the competitiveness of the software market and their changing technologies. In software systems with highly volatile requirements, traceability has become a critical issue. Numerous researchers have put their work over past years on traceability from problem space to solution space in traditional software development and evolution [13,47-49]. In this regard, today there are still several challenges to be dealt with. Specifically the Center of Excellence for Software Traceability identified eight challenges related to the purpose, cost, configuration, confidence, scalability, portability, value, and ubiquity of traceability [20]. This traceability is even more challenging in recent software development paradigms such as Software Product Line Engineering (SPLE $[14,46]$ ). In fact, the capability of trac- 
ing variability in a family of the products is still a challenge [37], as well as important: "the traceability work that is emerging from product line engineering contexts may have wider applicability to broader traceability reuse" [20].

This paper focuses on the traceability between the artifacts resulting from the SPLE phases domain analysis [26] and product-line architecting [35]. During the domain analysis phase, feature models [25] are usually used to describing requirements in terms of common and variable features of the set of products that make up a SPL. Then, these features are realized and described at architectural level in product-line architecture ( $P L A)$ models. Our approach is somewhat based on the work by Ramesh \& Jarke [49] and Pohl et al. [47], but particularizes the traceability definition between requirements and architecture in features and PLA by taking into account the traceability of variability. This traceability of variability is critical to configure the PLA and realize the products while ensuring that they meet the requirements, i.e. to check that the variability binding performed during the configuration of products satisfies the product requirements.

How variability is specified in feature and PLA models largely determines how variability can be traced. There has been an extensive research on supporting the representation of variability in feature models [25, $5,9]$, PLA models $[23,43,6,16,60,1]$, as well as those approaches that propose dedicated variability models [46, $7,30]$. Based on this state-of-the-art, current SPL traceability mechanisms trace the existing variability in feature models to variations in the PLA. This traceability is usually related to variations in the configuration of architectures as well as in the configuration of composite components [31], aka. subsystems [24]. These variations are realized through adding or removing components and/or connectors. This means, the configuration of architecture is customized by selecting optional, alternative, or multiple components and their respective connectors. We refer to these kinds of variations as external variability.

However, external variability is not enough to completely define all kinds of variations [6] and to trace them from features to PLA [60]. This happens when variations have a lower-granularity than the granularity of components (e.g. classes, services, or interfaces that implement functionalities such as logging, database connections, listeners of an event-based architecture, graphical controllers, etc.), so that they are materialized inside simple components - or non-composite components. In these components, in which variability occurs inside, part of their functionality is common to the SPL and part of their functionality changes de- pending on the product to be realized. As a result, in order to support this internal variability, it is necessary to specify variations that are internal to components. We refer to this kind of variations as internal variability. In addition, this internal variability is especially relevant, but no specific, when describing variability that refers to non-functional features or quality attributes [32], since they may crosscut several components of the PLA. For example: Suppose an illustrative example of a SPL for banking systems that consists of a set of core components that offer their functionality to Automatic Teller Machines (ATM) and bank web applications (WebApp). Both ATM and WebApp aim to provide a cost-effective service to bank customers that is convenient, safe, and secure 24-hour access for realizing a common set of banking transactions. A few lines of code implementing the functionality regarding quality attributes, such as availability or data encryption, are necessary. This code is scattered across the components WebApp and ATM and it has variations in its behavior depending on the specific banking system product by selecting strict or non-strict availability or different encrypting algorithms. Therefore, this internal variability could affect many different products or there could even be conflicting quality attributes (e.g. trade-offs between availability and performance) in different products of the same family. As a result, the absence of traceability that considers internal variability implies that it is not possible to check if the SPL products with internal variability meet the requirements. Therefore, the capability of tracing internal variability is as important as the capability of tracing external variability.

This paper presents a solution to trace variability from features to both external and internal architectural variability. This solution has been constructed using the metamodeling approach, since models automate development tasks and stimulate learning and reasoning capabilities, which is essential for tracing artifacts. Therefore, our solution is constituted by a set of models for describing and tracing PLAs from features. The description of features is supported by the Feature Model $[25,15]$. The description of PLAs is supported by a previous work that presents the FlexiblePLA Model [45] as a solution for specifying both (i) external variability of the architecture configuration and composite components, and (ii) internal variability of simple components. Specifically, in this paper we present a model that supports traceability between features and PLA, called Feature-PLA Traceability Model. The Feature-PLA Traceability Model defines the principles that govern the traceability links between the Feature model and the Flexible-PLA model, i.e. the rules that must be met to create links between the two mod- 
els. These rules assist software engineers in defining both coarse-grained and fine-grained links which trace external and internal variability between features and PLA. The goal of also tracing internal variabilityi.e. at fine-grained level - is to reduce error-prone decisions at the time of configuring variability to derive products - from a SPL platform - according to productspecific requirements. The usage of the Feature-PLA Traceability Model is possible due to the FPLA modeling framework ${ }^{1}$.

We have put the Feature-PLA Traceability Model into practice in a software factory, in a project for developing a product line of power metering management applications for Smart Grids. Validation is performed using the case study technique following the guidelines of Runeson and Höst for describing case studies [51]. This case study allowed us to obtain evidence of that the Feature-PLA Traceability Model was effective and helped engineers in the development and configuration of a successful product line in an industry project.

The structure of the paper is as follows: Section 2 describes background in which our solution is based on. Section 3 describes the Feature-PLA Traceability Model. Section 4 presents the case study used to validate the Feature-PLA Traceability Model, i.e. its viability, effectiveness, and helpfulness in an industry project. This section also discusses about limitations of our solution. Section 5 analyzes related work. Finally, conclusions and further work are presented in Section 6 .

\section{Background}

This section describes the required background to detail the contribution of this paper, i.e. the models that the Feature-PLA Traceability Model traces.

\subsection{The Feature Model}

Numerous methods for domain analysis can be found in literature, although one of the most widely used is the Feature-Oriented Domain Analysis (FODA) [25,5] in which our work is based on. The FODA method introduces the feature modeling technique for capturing commonality and variability of SPL in terms of features. This method defines a feature as "a prominent or distinctive user-visible aspect, quality, or characteristic of a software system or system" [25]. Feature modeling is graphically described through the feature diagram notation, which specifies all products of a family through a hierarchical tree-like structure. We use the extended

\footnotetext{
1 It is available on: https://syst.eui.upm.es/FPLA/home
}

feature metamodel definition proposed by Czarnecki et al. [15] which includes the following concepts:

- A root feature modularizes the model in a tree-like structure, in which there is a main root.

- Solitary features represent mandatory or optional characteristics of a software system which can be composed of zero or more solitary features and by zero or more feature groups.

- A feature group consists of a set of grouped features which in turn can be composed of zero or more solitary features and by zero or more feature groups. Feature groups can be $O R$ or $X O R$. The first one forces to choose $m$ grouped features (being $\mathrm{m} \leq$ total number of grouped features). The second one forces to choose only one grouped feature.

Figure 1 exemplifies these concepts through a simple feature model of a family of e-readers. The root feature of the tree is called e-readers family. A set of solitary features are hooked to the root, such as interface and connectivity. The solitary feature interface is composed of a XOR feature group that supports customized interfaces, such as keyboard or multi-touch, whereas the solitary feature connectivity is composed of the solitary features $W i-F i$ and $3 G$. The solitary feature $W_{i-F}$ is a mandatory feature for all products of the e-readers family while $3 G$ is optional.

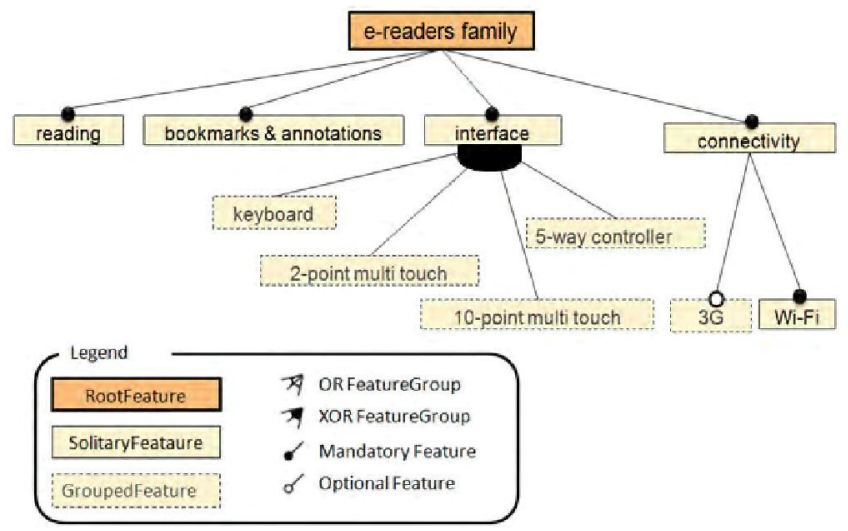

Fig. 1 E-readers SPL: Feature Model

\subsection{The Flexible-PLA Model}

The Flexible-PLA Model [45] is a precise representation for capturing variability as part of PLAs. The main concept underlying Flexible-PLA Model is the concept of Plastic Partial Component (PPC [45]). The concept of PPC is a solution to completely support the internal variation of architectural components. Therefore, it 
is a component that part of its behavior corresponds to the core of a SPL and part of its behavior is specific of a product or set of products from that SPL. The other concepts that are common to PLAs, such as components, connector, ports, etc., are specified as it is usually done in common Architecture Description Languages [36].

The variability mechanism underlying PPCs is based on the principles of invasive software composition and the combination of two approaches to define software architectures: the Component-Based Software Development [58] and the Aspect-Oriented Software Development [27]. The variability of a PPC is specified using variability points which hook fragments of code to the PPC known as variants, and weavings which specify where and when extending the PPCs using the variants. Weavings are defined outside from PPCs and variants so that these PPCs and variants are independent of the weaving or linking context. As a result, variants can be reused and crosscut several PPCs of the PLA. Additionally, PPCs reduce dependences and coupling between components and their variants, and enable easy and cheap (un-)weaving of variants. These advantages have been successfully applied to SPLs $[45,44,17]$.

The concepts of the Flexible-PLA model are exemplified by the graphical representation of a PPC called interface (see Figure 2). The PPC interface defines a variability point which hooks the code that implements the variants keyboard and multi-touch.

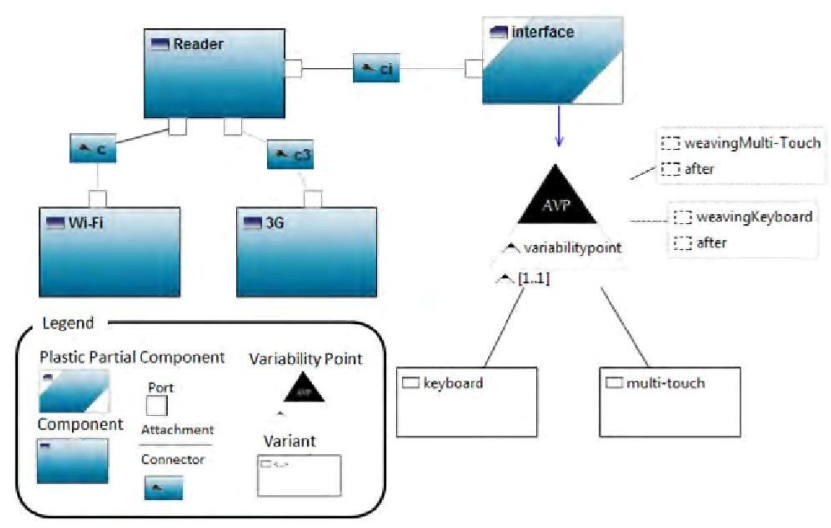

Fig. 2 E-readers SPL: Flexible-PLA Model

\section{Feature-PLA Traceability Model}

This section presents the Feature-PLA Traceability Model as a solution for tracing features to PLA. Tracing artifacts aims to automate development tasks, as well as to stimulate learning and reasoning. Models, traceabil- ity between models, and model transformations are the basis to automate development tasks, which is known as model-driven development (MDD [11]). Additionally, models help us understand complex problems and their potential solutions through abstraction [55], and could stimulate learning and reasoning [56]. For this reason, the proposed solution is based on models, specifically the Feature and Flexible-PLA Models, as well as the Flexible-PLA Traceability Model that defines traceability between the two first models.

The Feature-PLA Traceability Model provides modeling primitives to define traceability links, i.e. relationships, between elements belonging to the Feature Model (see Section 2.1) and elements belonging to the Flexible-PLA Model (see Section 2.2). These relationships are established between the set of feature elements and a set of architecture elements that satisfy them (aka. Satisfaction Links [49]). Hence, a feature element may define some kind of constraint or goal which may be satisfied by one or more architecture elements, while an architecture element may satisfy one or more feature elements. In this regard, the Feature-PLA Traceability Model defines the rules that govern the creation of these relationships. These rules are called linkage rules.

To be able to use these modeling primitives, it is necessary to define a domain-specific (modeling) language (DSL [59]). The next subsections describe (i) a DSL abstract syntax through the definition of the Feature-PLA Traceability metamodel, its domain concepts, relationships and rules, (ii) a DSL concrete syntax by defining a graphical language representation, and (iii) how putting these modeling primitives in practice.

\subsection{Abstract syntax: metamodel description}

Metamodels describe how models can be specified and establish the properties of models in a precise way. In addition, a metamodel is characterized because it allows the verification of those models that are constructed and conformed to it [12]. The realization of MDD principles is made around a set of OMG standards like MOF [40] which is a metametamodel. Specifically, our solution is based on MOF 2.0 and uses UML 2.0 to specify a metamodel which we refer to as Feature-PLA Traceability metamodel.

The Feature-PLA Traceability metamodel (see Figure 3 ) is composed of a set of inter-related metaclasses. These metaclasses define a set of properties and services for each concept considered in the model. On the one hand, metaclasses, their properties and their relationships describe the structure and the information that is necessary to define traceability links and their linkage rules. On the other hand, the services of metaclasses 


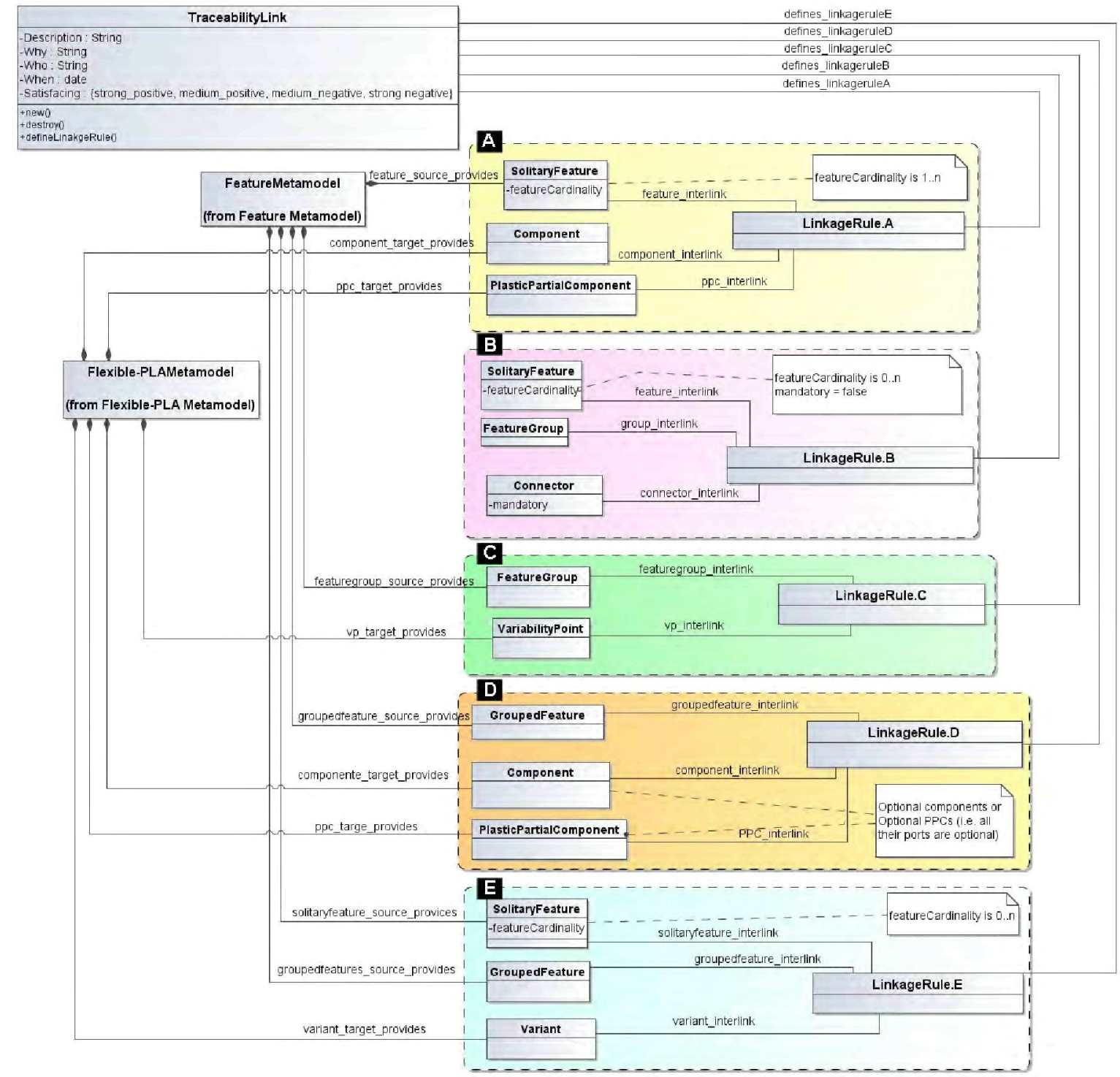

Fig. 3 Feature-PLA Traceability metamodel

offer the primitives to develop instances by creating, destroying, adding or removing elements which are compliant with the constructors of the metamodel ${ }^{2}$. Those constraints that cannot be defined through the use of relationships and their cardinality are specified by using the Object Constraint Language (OCL [41]), such those described as textual information in UML notes (see Figure 3).

The Feature-PLA Traceability metamodel is created with the aim of facilitating its integration with general-purpose traceability metamodels, such as the Metamodel for Requirements Traceability [29], or the EML Trace [28]. These models define the concept of traceability link through a metaclass that supports the traceability between any two models. We have re-used

2 Most services are omitted to gain readability. this metaclass, hence traceability links are described by the metaclass TraceabilityLink (see Figure 3). It offers the primitives to instantiate traceability links. The metaclass TraceabilityLink has five attributes (see Figure 3). Additionally, in order for the user to set the traces between the right elements, it is necessary to define a set of linkage rules that establish the constraints that govern the creation of these traces. To that end, the metaclass TraceabilityLink defines five linkage rules (see dashed rectangles labeled from A to E in Figure 3). Attributes and linkage rules as described below.

The metaclass TraceabilityLink has the attributes Description, Why, Who, When and Satisfacing. These properties store semantic knowledge about the traceability links. The attribute description keeps a brief description of the link. The attribute why stores the 
traceability link's rationale. The attributes who and when keep who creates the traceability link and when it is created, respectively. The definition of the attribute satisfacing is based on the work by Ramesh \& Jarke [49] who defined a scheme for assigning qualitative degree of satisfaction to links, i.e. a measure of the extent of how long one element affects another. Hauser \& Clausing [21] use four categories to relate how design affects quality requirements: strong positive, medium positive, medium negative, and strong negative. Positive values measure the degree to which features are satisfied, e.g. a recovery feature to provide response of 100 millisecond may be considered to be well satisfied, so that 90 millisecond and 110 millisecond response time may be considered to satisfy the feature with different degrees. Negative values may capture trade-offs between features, e.g. a component that satisfies an availability feature may have a strong negative impact on a performance feature. This scheme is incorporated in our traceability model as follows: an element belonging to the Flexible-PLA Model may contribute toward satisficing an element belonging to the Feature Model along these four categories. Thereby, it is possible to assign the values strong positive, medium positive, medium negative, and strong negative to the attribute satisficing of a traceability link.

The metaclass TraceabilityLink defines five linkage rules (see the association relationships between the metaclass TraceabilityLink and the metaclasses LinkageRule. $\{\mathrm{A}-\mathrm{E}\}$ in Figure 3 ). The linkage rules define how relationships can be established, i.e. the rules that restrict which elements belonging to the Feature Model can be traced to which elements belonging to the Flexible-PLA Model. These linkage rules act as constraints that must take variability into account. Variability in the Feature Model is specified by means of optional solitary features, feature groups, and grouped features. Variability in the Flexible-PLA Model is specified by means of optional components and optional connectors, which describe external variability of architecture configuration, as well as variability points and variants, which describe the internal variability of PPCs. These forms of variability constrain the traces that can be defined in such a way that the linkage rules define the following constraints:

Linkage Rule A: A mandatory solitary feature can trace to a component or a PPC.

Linkage Rule $B$ : An optional solitary feature can trace to an optional connector. A feature group can trace to an optional connector.

Linkage Rule $C$ : A feature group can trace to a variability point.
Linkage Rule D: A grouped feature can trace to an optional component or an optional PPC.

Linkage Rule E: An optional solitary feature can trace to a variant. A grouped feature can trace with a variant.

These constraints of the traces are implemented in the Feature-PLA Traceability metamodel through five metaclasses to which we refer to as LinkageRule. $\{A-E\}$ (see Figure 3). These metaclasses define associations with the metaclasses from Feature and Flexible-PLA metamodels ${ }^{3}$. As a result, any link between an element from a Feature model and an element from a FlexiblePLA model must be compliant with one of these linkage rules. As the linkage rules support external and internal variability, both fine-grained and coarse-grained traceability links can be defined.

In this regard, it is necessary to highlight that we decided not to add a new attribute (with the types of linkage rules and define the corresponding OCL constraints) into the metaclass TraceabilityLink in order to preserve the metaclass TraceabilityLink of generalpurpose traceability models. In order to realize this typing we defined five linkage rules through five metaclasses. In this way, we guarantee that the Feature-PLA Traceability Model can be reused by and integrated in other traceability models.

Finally, it is necessary to highlight that this metamodel conforms to a general-purpose Traceability model (see [18]) which is located in an upper layer of the MOF Architecture [40] (meta-metamodel layer). In this way, the metaclasses TraceabilityLink and LinkageRule. $\{A-$ $E\}$ conform to two meta-metaclasses (TraceLink and LinkageRule) of this meta-metamodel. The definition of the metaclasses LinkageRule. $\{A-E\}$ allowed us to extend the rationale of the linkage rules.

\subsection{Concrete Syntax: Graphical language description}

A graphical modeling language has been defined as this kind of languages is usually more intuitive.

Figure 4 illustrates the Feature-PLA Traceability graphical language through an example of a SPL of ereaders. Figure 4 shows six traceability links and their properties - satisfacing, who, and when (see ID_001 to ID_006). The traceability link ID_003 defines a relationship between the optional solitary feature $3 G$ and the optional component that implements it (e.g. see the properties: strong positive, J.Smith and 03/09/2012). This link traces a variation that is materialized by adding

3 The Feature metamodel is described in [15] while the Flexible-PLA metamodel is described in [45] 


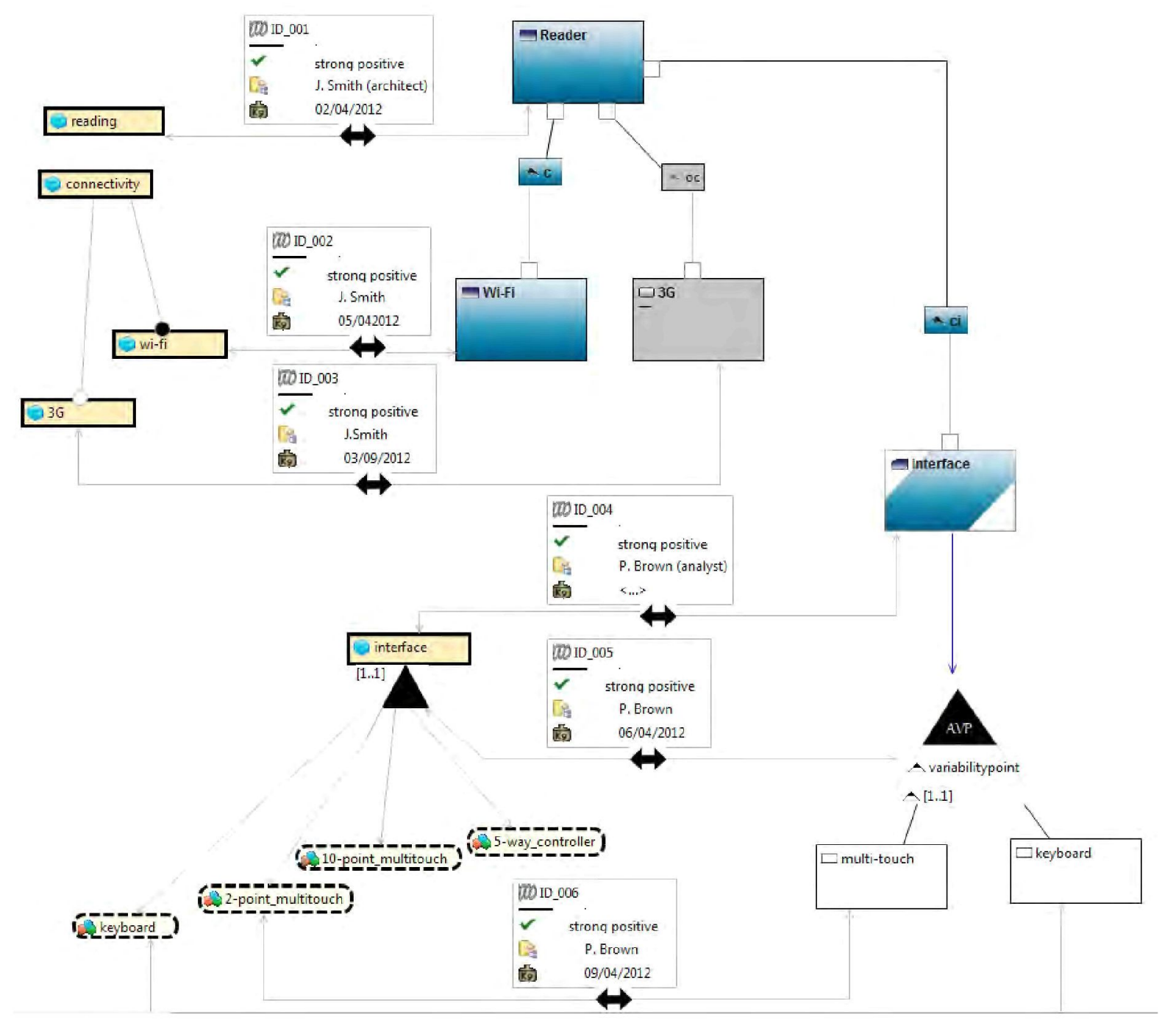

Fig. 4 E-readers SPL: Feature-PLA Traceability Model

or removing the component $3 G$ to/from the configuration of the PLA. Therefore, this link traces external variability. The traceability link ID_005 defines a relationship between a point of variability related to the types of interfaces - a feature group - and the variability point that implements it. Finally, the traceability link ID_006 defines a relationship between the grouped feature 2-point multitouch and the variant that implements it. This link traces a variation that is not materialized by adding or removing a component because of its small size - it is a service called listenTouch (see Figure 5). This variation is materialized by weaving or unweaving the variant multi-touch to/from the PPC interface, i.e. by injecting (or not) the service listenTouch instead, before, or after the execution of the service listenAction of the PPC interface. Therefore, this link traces internal variability.

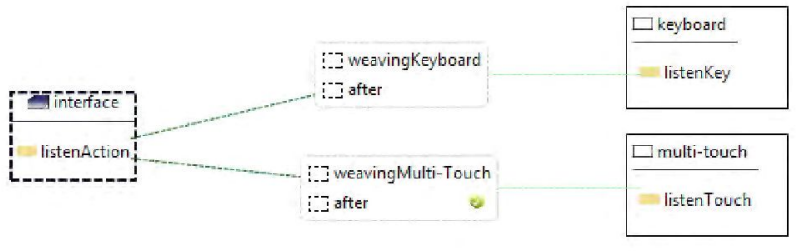

Fig. 5 E-readers SPL: weaving definition

\subsection{Feature-PLA Traceability Model in Practice}

The solution presented in this paper for tracing variability from features to PLA is supported by the FPLA modeling framework. FPLA is an open-source graphical tool that is available for the community as an Eclipse plug-in ${ }^{4}$. The use of the FPLA modeling framework to put this solution into practice is described through a set of activities as follows.

\footnotetext{
4 https://syst.eui.upm.es/FPLA/home
} 
1. SPL domain analysts model the problem space, i.e. specify common and variable features through a Feature model.

2. SPL architects model the solution space (PLA), i.e. specify the PLA structural configuration through a Flexible-PLA model.

3. Both domain analysts and architects define the traceability links between a Feature model and a FlexiblePLA model, i.e. establish the relationships between elements from these two models through a FeaturePLA Traceability model.

4. SPL developers implement and test the components and services of the SPL. The resulting source code is linked to the components specified in the FlexiblePLA model. To do this, Flexible-PLA models provide links to external sources.

5. Product engineers configure specific products through the binding of the variability according to the product needs - product-specific requirements. The FPLA modeling framework allows product engineers to specify this binding (see the $\checkmark$ mark in Figure 5 that selects the multi-touch feature).

6. Product engineers examine the Feature-PLA Traceability model to ensure that the variability binding was correctly performed. This means, to check that the binding performed in the PLA meets and satisfies the product-specific requirements.

7. Finally, the FPLA modeling framework automatically binds the variability from PLA to code in order to configure components and generate code for specific products. This means, FPLA automatically generates code skeletons from Flexible-PLA models and composes the code from external sources by using model-to-text transformations.

\section{Case Study}

This section aims to provide empirical evidence that validates that the use of the Feature-PLA Traceability Model is viable in an industry project, as well as effective and helpful for developing and configuring software product lines. Since the goals to be validated are qualitative, we use the case study technique. Case study research is a technique that consists of the investigation of contemporary phenomena in their natural context [61] to search for evidence, gain understanding, or test theories by primarily using qualitative analysis [50].

The case study was conducted in an experimental ismart software factory (iSSF [33]) which is deployed in the Technical University of Madrid $\left(\mathrm{UPM}^{5}\right)$ and Indra

5 http://www.upm.es/internacional
Software Labs ${ }^{6}$. Specifically, the case study was performed within an industrial project on Smart Grids [34] to develop a SPL of a family of power metering management systems. The authors of this paper have been involved since 2011 with this particular investigation.

The iSSF is a software engineering research and education setting in close cooperation with the top industrial and research collaborators in Europe. It is a global and distributed software development initiative set up at the end of 2011. Indra Software Labs leads this initiative at the corporate level in Spain, in conjunction with UPM, although it is framed into a broader-scope that includes other software factories such as that located at the Univ. of Helsinki, Univ. of Eastern Finland and Univ. of Bolzano and companies such as Tieto, and Indra in Spain. This initiative aims to put in practice models and tools that will contribute both toward the implementation of the new processes and methodologies, and the monitoring and tracking of the results.

The iSSF in which the case study has been run, comprises laboratories in two different geographical locations in Madrid (UPM and Indra's factories), equipped with sophisticated computer and monitoring equipment. This equipment facilitates tracking of the project's progress using real-time data from development tools. The iSSF facility continuously runs projects in sixteen week cycles. Therefore, it is a suitable setting to deploy, track and evaluate the applicability of the Feature-PLA Traceability Model.

Next, the case study is reported according to the guidelines for conducting and reporting case study research in software engineering by Runeson and Höst [51]. The goal of reporting a case study is twofold: to communicate the findings of a study, and to work as a source of information for judging the quality of the study. With this twofold goal, the reporting of the case study is described as follows.

\subsection{Case study design}

This section describes the case study, the research objective and questions, the data collection procedure, analysis and validation procedures, and the subjects participating in the case study.

\subsubsection{Research objective and questions}

Evidence of the viability of the Feature-PLA Traceability Model can be obtained by putting the model into practice in a real life setting. Therefore, the research objective focuses on evaluating the effectiveness

\footnotetext{
6 http://www.indracompany.com/en
} 
of the Feature-PLA Traceability Model as well as the helpfulness that it could provide SPL engineers. The criterion to validate the achievement of the objective is defined as the capabilities to (i) trace both coarsegrained and fine-grained variability in order to satisfy the traceability of most common kinds of variations, and (ii) provide knowledge to help SPL engineers at the time of configuring the different products that make up a SPL, i.e. when variability has to be bound according to the product-specific requirements. Hence, the research questions to be answered through the case study analysis can be formulated as follows:

$R Q_{1}$ : Are Feature-PLA Traceability modeling primitives effective in providing SPL engineers the means for specifying traceability for most common kinds of variations that they define on their product family? $R Q_{2}$ : Do Feature-PLA Traceability models assist and guide SPL engineers at the time of configuring products of their product family?

Research question $R Q_{1}$ aims to find out if the FeaturePLA Traceability Model effectively provides SPL engineers with mechanisms to trace all types of variations that they commonly define, which includes the capability of tracing those features that are realized through external variations of the PLA configuration (i.e. traceability of external variability) as well as the traceability of those features that are realized through internal variations of the components (i.e. traceability of internal variability). The level of effectiveness is a dependent variable, i.e. a variable of interest for being analyzed and evaluated. It is measured in terms of the percentage of variations existing in the domain of the SPL under study (i.e. variations in the Feature model) that can be traced by using the modeling primitives provided by the Feature-PLA Traceability Model. The potential independent variables that might have an influence on the dependent variable are the project size, the $S P L$ domain, the complexity of Feature and PLA models, and the total number of variations identified in the product family.

Research question $R Q_{2}$ aims to find out if the knowledge stored in Feature-PLA Traceability models is really helpful for SPL engineers at the time of configuring the products of their product family. In this regard, helpfulness is defined in this paper as the facilities provided for engineers to enable product configuration (i.e. selection of variants and construction of product applications). As a dependent variable, the level of helpfulness to configure products is qualitatively estimated by analyzing questions asked to the SPL engineers involved in the cases study through a set of interviews. These questions asked the SPL engineers about specific situa- tions in which the assistance of Feature-PLA Traceability models to configure products was analyzed. Hence, the SPL engineers were asked if Feature-PLA Traceability models helped them when trying to bind variability to configure specific products from the product family, while ensuring the product requirements compliance. The potential independent variables which might have an influence on the dependent variable are the engineers experience, the project size, the PLA complexity, the misinterpretation of interview questions and the total number of variations identified in the product family.

It is necessary to mention that it is in the nature of case studies that independent variables cannot be controlled [50]. This and other potential threats to validity are discussed in subsection 4.2.3.

\subsubsection{Data collection procedure}

In the case study, we have gathered both quantitative and qualitative data. The collection methods which have been used are the following:

- Observation. Two observers attended project meetings and visited the team twice a week. They took notes from these meetings and, thanks to the iSSF technologies, meetings were video recorded, transcribed, and analyzed using the constant comparison method as described in [22].

- Questionnaire and Interview. Stakeholders were interviewed following a questionnaire ${ }^{7}$ open to the discussion. These interviews were video recorded, transcribed, and analyzed using the constant comparison method.

- Archival data. In addition to the storage of the video recordings, the information about the project was collected in Redmine ${ }^{8}$

- Analysis of work artifacts. Feature-PLA Traceability models generated with the FPLA modeling framework were gathered.

\subsubsection{Analysis \& Validity procedure}

In this case study, both quantitative and qualitative analysis were used to examine the data gathered. For quantitative data, this case study uses analysis of descriptive statistics. For qualitative data, the procedure to explore the chain of evidence [50] from collected data is described as follows: Interviews and meetings are recorded, transcribed, grouped by quotes and coded. Coding means that parts of the text are given a code

7 The script of the interviews is available on https://www.surveymonkey.com/s/TSYCCN6

8 Redmine is web-based project management and bugtracking tool http://www.redmine.org/ 
representing a certain topic of interest - one code is usually assigned to many pieces of text, and one piece of text can be assigned more than one code and codes can form a hierarchy of codes and sub-codes [50]. The coded material is enriched with comments and reflections (i.e. memos). From this material it is possible to identify evidence that answers the research questions.

As data gathered in case studies is mainly qualitative [50], and it is typically less precise than quantitative data, it is important to use triangulation to increase the precision of the study. There are several types of triangulation [57]: (i) methodological triangulation, i.e. the use of different methods to measure the same concern; (ii) data source triangulation, i.e. the use of multiple data sources at potentially different occasions; and (iii) observer triangulation, i.e. the use of more than one observer in the case study [22]. In order to increase the precision of the qualitative analysis and its obtained results, the three types of triangulation were used in this case study. Methodological triangulation was performed through interviews, observations, and the analysis of archival data. Data source triangulation was performed by interviewing the SPL engineers both separately and together. Finally, observer triangulation was applied by replicating specific data collection sessions by two different observers.

\subsubsection{Case study description}

The case study consists of a project to model, design, and implement a "power quality monitoring and a remote control and smart metering" platform. It is part of two larger ITEA2 projects called IMPONET ${ }^{9}$ (127 man-years) and NEMO\&CODED ${ }^{10}$ (112 man-years), and a third national project called ENERGOS ${ }^{11}(24,3$ million Euros). These three projects focused on supporting complex and advanced requirements in energy management, specifically in electric power networks that are conceptualized as Smart Grids [34]. Smart Grids are composed of an aggregation of a broad range of energy resources, from large generating systems (traditional sources, e.g., nuclear power plants, hydro power plants) to smaller generating systems (called microsources, e.g., small solar farms, distributed wind generators),

\footnotetext{
9 Intelligent Monitoring of Power NETworks http://www.itea2.org/project/index/view?project $=10032$

10 NEtworked MOnitoring \& trol, Diagnostic for Electrical Distribution http://www.itea2.org/project/index/view?project $=1131$

11 Technologies for automated and intelligent management of power distribution networks of the future http://www.indracompany.com/sostenibilidad-einnovacion/proyectos-innovacion/energos-technologies-forautomated-and-intelligent-
}

Remote control \& Smart Metering Platform

\begin{tabular}{|l|c|c|}
\hline Meter Data Management & \multicolumn{2}{|c|}{ End-user Access Platform } \\
\hline Head End & Smart Metering & Data Exchange \\
\hline
\end{tabular}

Power Quality Monitoring

Communication Platform

Fig. 6 Modules of the power quality monitoring and the remote control and smart metering platform

operating as a single system providing both power and heat [34]. Smart Grids promote the integration of renewable energy resources and their distributed, open, and self-controlled nature.

The power quality monitoring and the remote control and smart metering platform is a software intensive software composed of a set of coarse-grained modules: Communication Platform, Power Quality Monitoring, Meter Data Management, End-user Access Platform, Head End, Smart Metering, and Data Exchange (see Figure 6). At this coarse-grained level, the platform presents variability related to the optionality of the module Power Quality Monitoring which depends on the grid, i.e. if the grid requires guarantee only the power supply or also power quality. Each one of these modules has multiple levels of decomposition and variability with different levels of detail that are briefly described below. This is why this project was envisioned as a SPL that allows configuring the platform depending on the Smart Grid requirements.

A representative example of the multiple levels of variability is the End-user Access Platform. This module is configurable by considering the following variants: type of GUI, end-user, and data. Regarding the type of GUI, the End-user Access Platform was designed to support Web application, Desktop application, Android application, as well as specific in-home device's application. Regarding the end-user, the functionalities and the information that are provided by the access platform to the end-users vary in the case of a distributor, a retailer, or a customer. This means that the information provided by the End-user Access Platform is variable depending on the end-user and the end-user requirements. Finally, the information that is shown in the GUI and the technologies used to display that information are variable depending on whether the data are provided in real-time or using historical data.

Other examples of multiple levels of variability are the Smart Metering and the Power Quality Monitoring modules. The first one implements a set of forecast- 


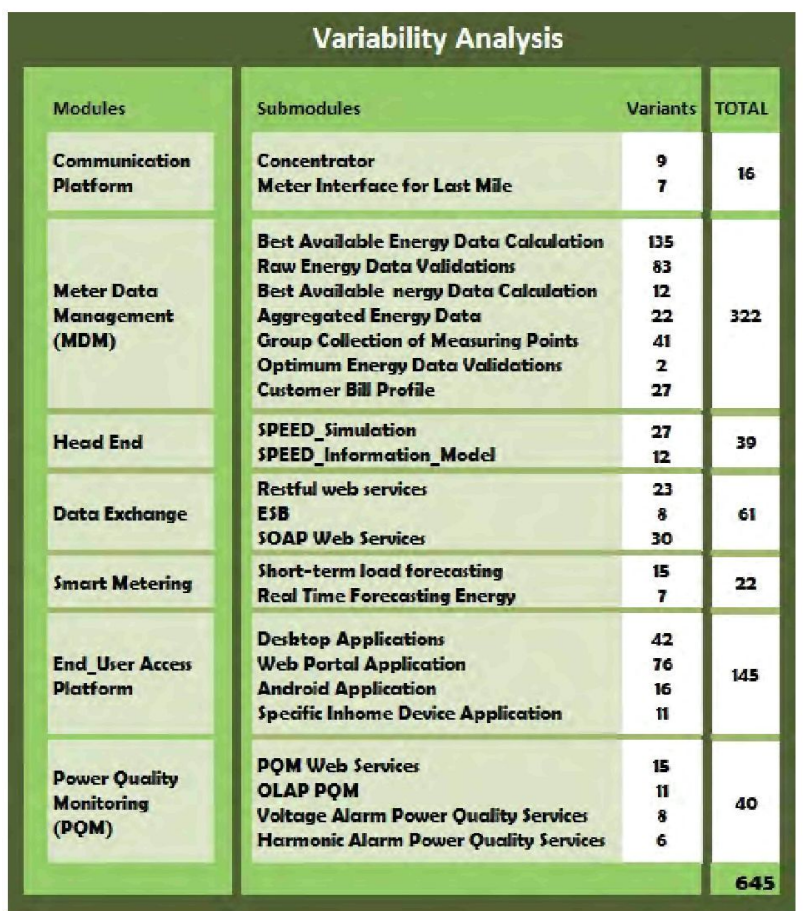

Fig. 7 The power quality monitoring and the remote control and smart metering platform Variability Analysis

ing algorithms that vary depending on the energy data used, for the next 24 or 48 hours, or whether it is calculated using real time energy data, historical energy data from the database, or both of them. The second one implements a set of power quality algorithms that can be configured in order to provide a variety of information, such as events, disturbances, alarms control, etc. Finally, the Communication Platform implements a Data Distribution Service (DDS [39]) based on the publication-subscription paradigm. This module is in turn a source of internal variability that crosscuts the other modules. Hence, DDS defines domains, partitions, and topics in order to specify different data space and organize the flow of data. The subscription to the topics is variable depending on, for example, the events or alarms to be controlled.

In order to illustrate the complexity of the system, and in particular the level of variability, the platform has more than 600 variants (see Figure 7 ). In this paper, we specifically report the part of the SPL that develops a family of power metering management systems for Smart Grids, i.e. the Meter Data Management module. This is due to space and understandability reasons. We refer to this part of the SPL as OPTIMETER SPL.

OPTIMETER SPL focuses on the development of a family of power metering management systems for Smart Grids (see the central box of Figure 8). A power metering management system captures and manages meter data from a large number of distributed energy resources. It validates, stores and processes these data, and provides them to external systems. Figure 8 shows an overview of a metering management system and its interaction with external systems to capture and provide meter data. The overview of the system functionality is as follows:

1. Meter capturing. This involves integrating all meter capturing processes (see Meter Capturer in Figure 8) which are currently being supported by telemetering systems and batch processes that collect measurements at substations (see box Input in Figure 8). The purpose is to have a single database with the energy metering data.

2. Meter processing. This includes three operations: the validation of meter data according to an established validation formula, the calculation of the optimal vector for a measuring point for a type and period of energy data, and the estimation of energy data according to a established estimation formula (see Meter Processor in Figure 8).

3. Meter providing. This involves defining the interface (see Meter Provider in Figure 8) with client information systems, such as billing and settlements, energy demand forecast, and energy purchases, to exchange data with them (see box Output in Figure 8).

Data processing should be done in real time. To do this, it is necessary to account for performance when loading the large amounts of energy data coming from the meter capturing processes as well as performance when querying these data. The OPTIMETER SPL aims to provide a family of systems, each of which is intended to support the different data storing technologies shown in Figure 9. The objective is to carry out various proof of concept of large data storing technologies to evaluate their performance. Therefore, the data storing technology is a variability point.

Meter providing should be available 24/7. Metering management systems should guarantee availability 24 hours 7 days per week of their core functionality to the external systems. Several applications require to have strict $24 / 7$ availability, while others permit a weaker, non-strict availability. Strict availability must provide recovery and repair in milliseconds, whereas non-strict availability is less available and cheaper. Therefore, the strictness of availability is another variability point.

The OPTIMETER SPL is being iteratively and incrementally developed in the iSSF in Scrum subprojects [53] of 8 iterations, aka. sprints (1sprint $=2$ weeks). This case study focuses on two of these Scrum subprojects which we refer to as Optimeter $I$ and Optimeter II. Optimeter I consisted of the development of 


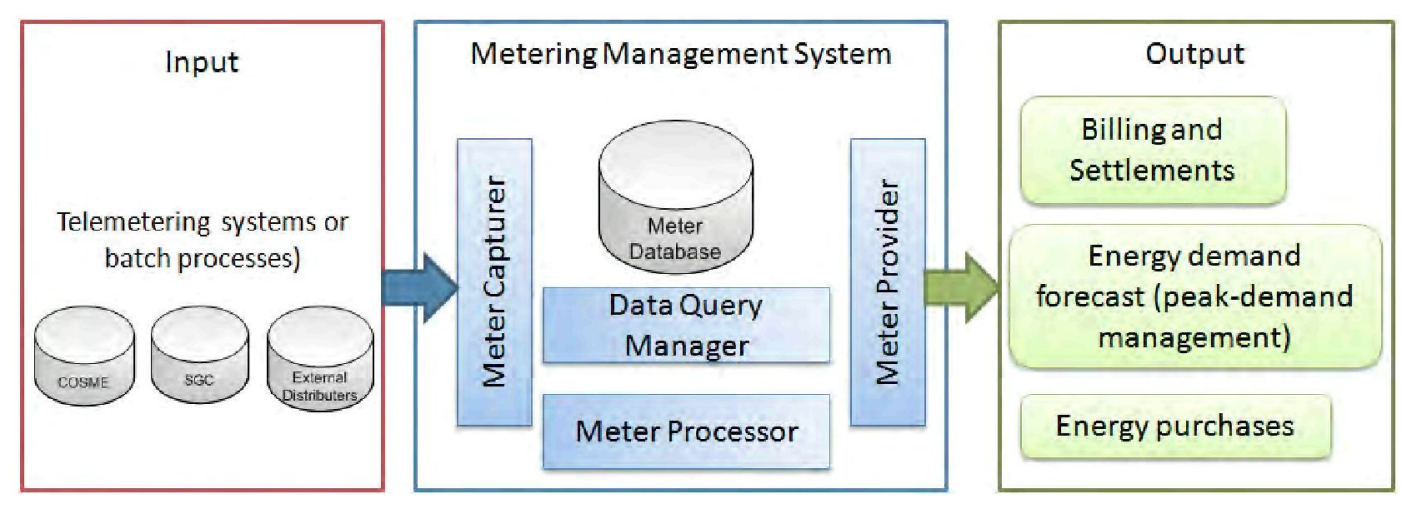

Fig. 8 Metering management system - An overview and interfaces with external systems

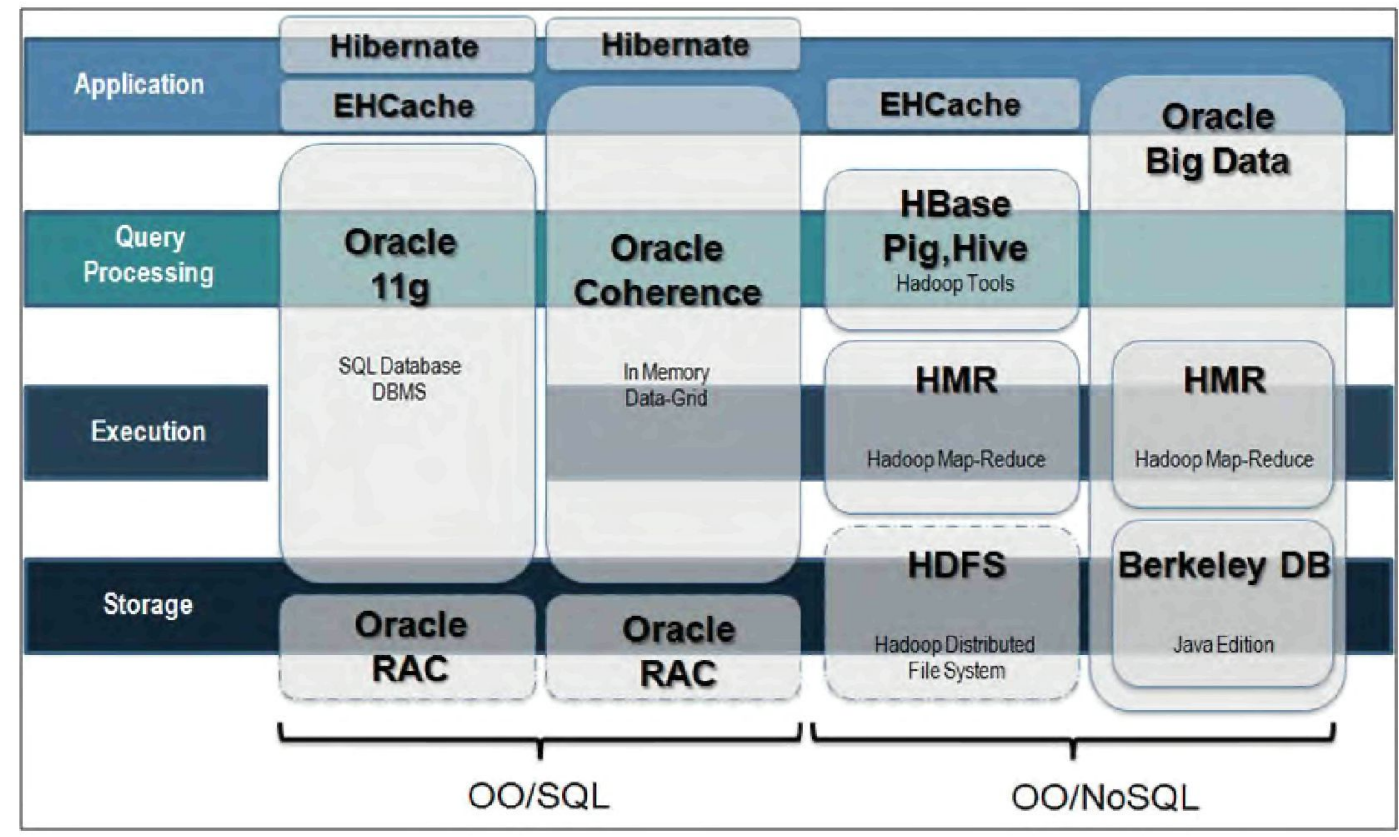

Fig. 9 OPTIMETER SPL - Evaluation of large data storing technologies for metering management systems

the OPTIMETER SPL platform from which a set of metering management system applications can be efficiently developed and produced (Domain Engineering [46]). Optimeter II consisted of the development of two of these product applications (Application Engineering [46]): a metering management application running over the Berkeley database ${ }^{12}$ and Hadoop clustering ${ }^{13}$ with strict availability, and a metering management application running over the Oracle $11 \mathrm{~g}$ data-

\footnotetext{
12 Oracle Berkeley DB is a high-performance embeddable database providing Java Object and Key/Value storage (NoSQL). http://www.oracle.com/technetwork/products/berkeleydb/

13 Apache Hadoop is a framework for running applications on large cluster built of commodity hardware. http://hadoop.apache.org/
}

base $^{14}$ and Oracle Real Application Clusters $(R A C)^{15}$ with non-strict availability (see Figure 9).

\section{1 .5 Subject description}

In total, 10 people participated in Optimeter I and II: four analysts/developers, two product owners, one scrum master (who performs both the tasks of the Scrum master and of a part-time architect), and one full-time architect. During the domain engineering - i.e. Optimeter $\mathrm{I}-$, the people involved in the project are referred in this case study as SPL engineers, while during the application engineering-i.e. Optimeter II-, the peopled are referred as product engineers. It is necessary

14 Object-relational database management system. http://www.oracle.com/technetwork/database/

15 Software for clustering and high availability in Oracle $\mathrm{db}$ environments. http://www.oracle.com/technetwork/products/clustering/ 


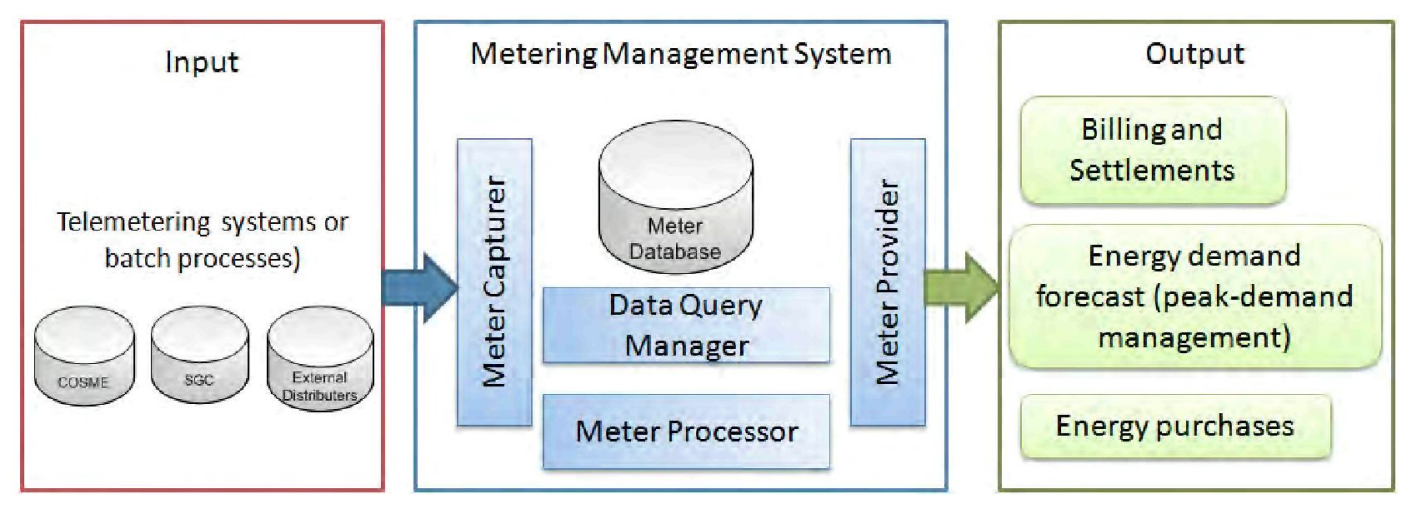

Fig. 8 Metering management system - An overview and interfaces with external systems

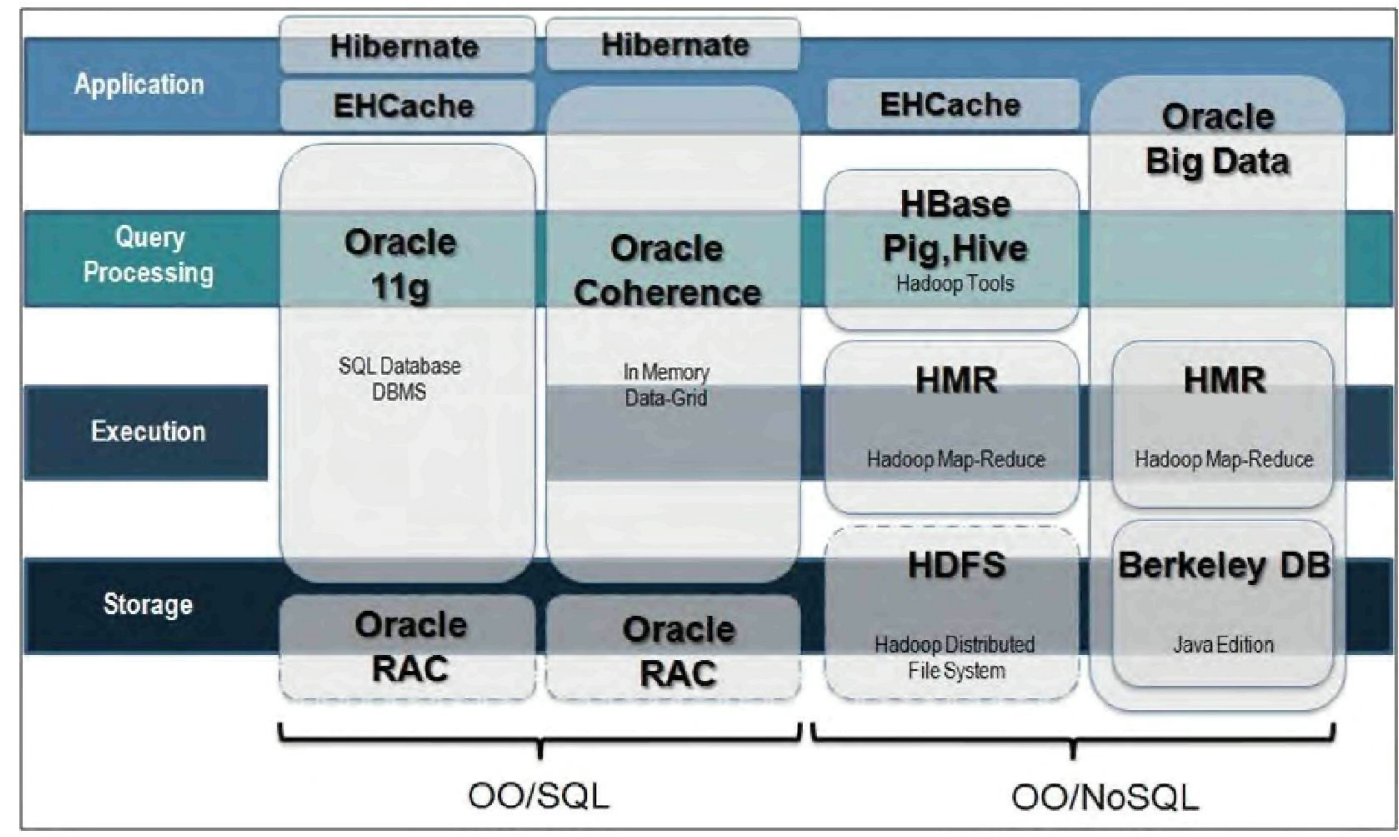

Fig. 9 OPTIMETER SPL - Evaluation of large data storing technologies for metering management systems

the OPTIMETER SPL platform from which a set of metering management system applications can be efficiently developed and produced (Domain Engineering [46]). Optimeter II consisted of the development of two of these product applications (Application Engineering [46]): a metering management application running over the Berkeley database ${ }^{12}$ and Hadoop clustering ${ }^{13}$ with strict availability, and a metering management application running over the Oracle $11 \mathrm{~g}$ data-

\footnotetext{
12 Oracle Berkeley DB is a high-performance embeddable database providing Java Object and Key/Value storage (NoSQL). http://www.oracle.com/technetwork/products/berkeleydb/

13 Apache Hadoop is a framework for running applications on large cluster built of commodity hardware. http://hadoop.apache.org/
}

base $^{14}$ and Oracle Real Application Clusters $(R A C)^{15}$ with non-strict availability (see Figure 9).

\section{1 .5 Subject description}

In total, 10 people participated in Optimeter I and II: four analysts/developers, two product owners, one scrum master (who performs both the tasks of the Scrum master and of a part-time architect), and one full-time architect. During the domain engineering - i.e. Optimeter $\mathrm{I}-$, the people involved in the project are referred in this case study as SPL engineers, while during the application engineering-i.e. Optimeter II-, the peopled are referred as product engineers. It is necessary

14 Object-relational database management system. http://www.oracle.com/technetwork/database/

15 Software for clustering and high availability in Oracle $\mathrm{db}$ environments. http://www.oracle.com/technetwork/products/clustering/ 


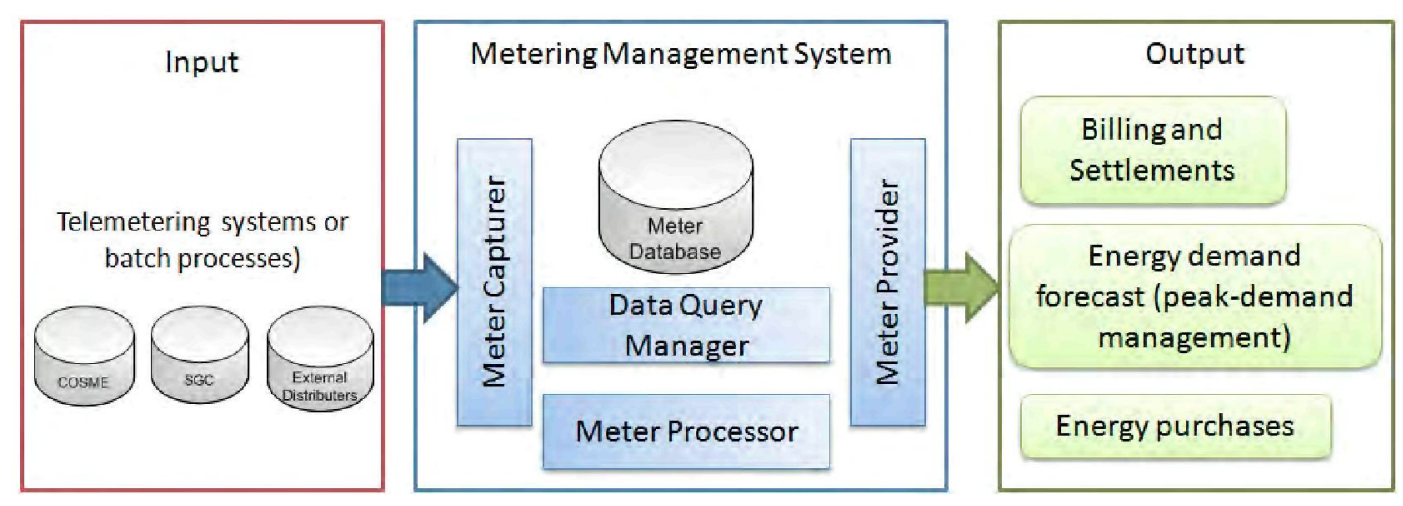

Fig. 8 Metering management system - An overview and interfaces with external systems

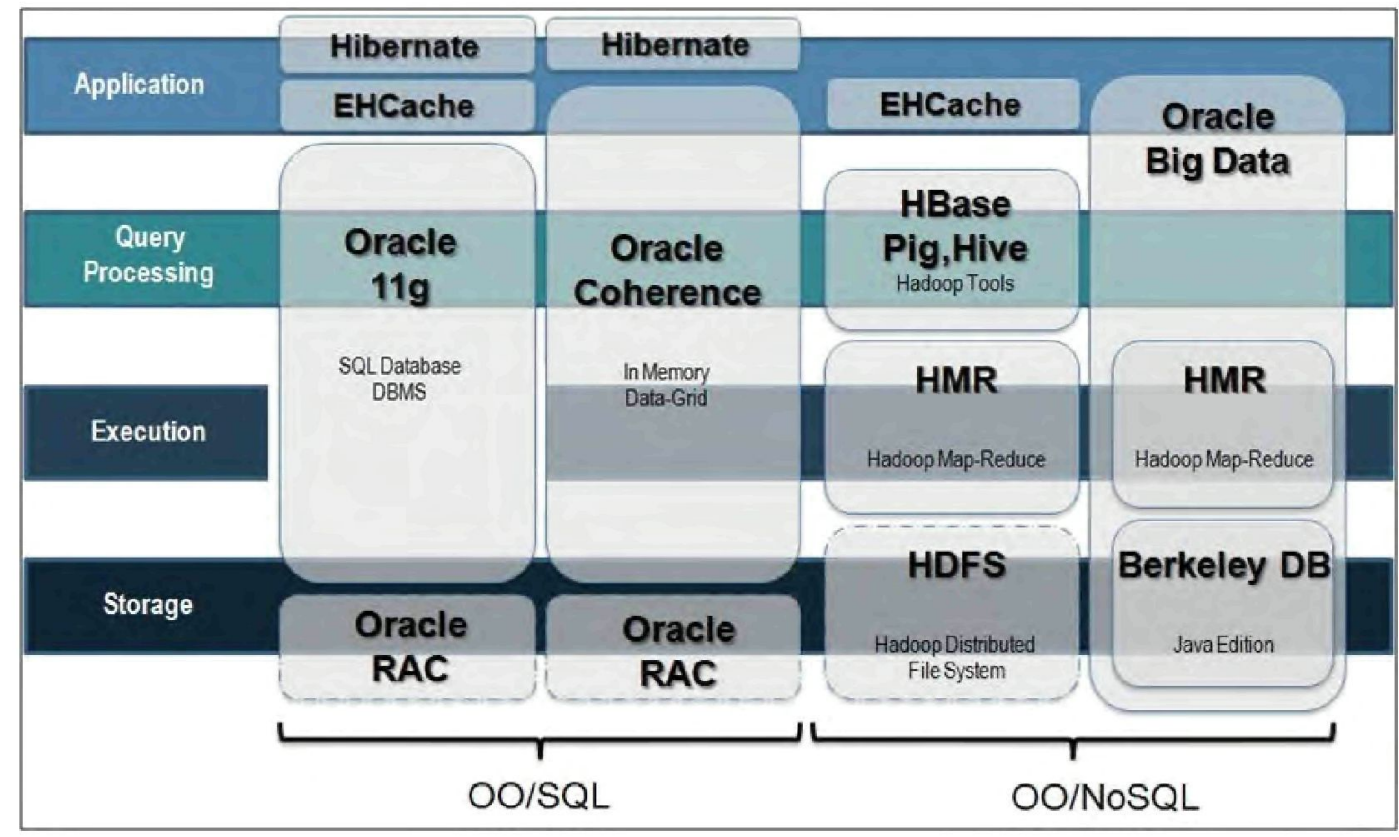

Fig. 9 OPTIMETER SPL - Evaluation of large data storing technologies for metering management systems

the OPTIMETER SPL platform from which a set of metering management system applications can be efficiently developed and produced (Domain Engineering [46]). Optimeter II consisted of the development of two of these product applications (Application Engineering [46]): a metering management application running over the Berkeley database ${ }^{12}$ and Hadoop clustering ${ }^{13}$ with strict availability, and a metering management application running over the Oracle $11 \mathrm{~g}$ data-

\footnotetext{
12 Oracle Berkeley DB is a high-performance embeddable database providing Java Object and Key/Value storage (NoSQL). http://www.oracle.com/technetwork/products/berkeleydb/

13 Apache Hadoop is a framework for running applications on large cluster built of commodity hardware. http://hadoop.apache.org/
}

base $^{14}$ and Oracle Real Application Clusters $(R A C)^{15}$ with non-strict availability (see Figure 9).

\section{1 .5 Subject description}

In total, 10 people participated in Optimeter I and II: four analysts/developers, two product owners, one scrum master (who performs both the tasks of the Scrum master and of a part-time architect), and one full-time architect. During the domain engineering - i.e. Optimeter $\mathrm{I}-$, the people involved in the project are referred in this case study as SPL engineers, while during the application engineering-i.e. Optimeter II-, the peopled are referred as product engineers. It is necessary

14 Object-relational database management system. http://www.oracle.com/technetwork/database/

15 Software for clustering and high availability in Oracle $\mathrm{db}$ environments. http://www.oracle.com/technetwork/products/clustering/ 
to highlight that the engineers involved in Optimeter I and II are not the same. Finally, two observers had access to all project information and collaborated directly with product owners and fellow team members.

\subsection{Results}

This section describes the execution, analysis and interpretation of the results from the case study execution, as well as the evaluation of its validity.

\subsubsection{Case study execution}

This section describes the execution of Optimeter I first, and then the execution of Optimeter II. These executions has been performed following the activities presented in subsection 3.3. The models resulting from these activities have been captured through snapshots from the FPLA modeling framework.

The first activity was feature domain analysis. Figure 10 shows the feature model that represents the features that OPTIMETER SPL must meet. The feature model has three points of variability - feature groupsthat implement different data storing technologies (database and clustering) and different degrees of availability. The feature model is described in detail as follows:

- F1_Meter Reading (see Figure 10) consists of reading metering data associated with different energy resources, periods (quarterly, hourly, daily, and monthly), and intervals.

- F2_Meter Storing (see Figure 10) consists of a large data store. There are two mutually exclusive alternative variations: one variant is Berkeley $\mathrm{DB}$ and the other variant is Oracle $11 \mathrm{~g}$ (see the grouped features Berkely $D B$ and Oracle11g in Figure 10).

- F3_Meter Data Accessing (see Figure 10) consists of initial data loading of historical metering data of one month and querying of these data. Both loading and querying require to leverage high performance through the use of clustering technologies. There are two mutually exclusive alternative variations: one variant is Hadoop clustering over Berkeley DB and the other variant is RAC over Oracle $11 \mathrm{~g}$ (see the grouped features Hadoop and $R A C$ in Figure 10).

- F4_Meter Data Processing (see Figure 10) consists of the algorithms for validating raw and optimal data, as well as calculating the optimal vector (integrated processing) of raw and optimal data. Namely, the energy data for a specific origin, period, and date is retrieved and the system adds data to obtain the energy data of the next period.
- F5_Meter Data Providing (see Figure 10) consists of an interface that provides metering data query to external systems.

- F6_Availability (see Figure 10). It ensures availability of metering data 24 hours 7 days per week. There are two mutually exclusive alternative variations: one variant implements strict availability and the other variant implements non-strict availability (see the grouped features strict and non-strict in Figure 10).

The second activity was product-line architecting. Regarding availability, various architectural tactics are proposed in the literature $[8,54]$. The SPL engineers selected active redundancy and passive redundancy tactics to implement strict and non-strict availability, respectively. These tactics are briefly described as follows.

- The tactic active redundancy is based on a "configuration wherein all of the nodes (active or redundant spare) in a protection group receive and process identical inputs in parallel, allowing the redundant spare(s) to maintain synchronous state with the active node(s)" [54]. Therefore, from the architectural view, this tactic requires: (i) a load balancer for all nodes - active and redundant nodes - to process identical inputs, and (ii) a synchronizer in order for the active and redundant nodes to maintain an identical state. If there is a failure, the repair occurs on time as the redundant spare has an identical state to the active node. The cost of this tactic is high due to the cost of synchronization between redundant spare and active node(s).

- The tactic passive redundancy is based on a "configuration wherein only the active members of the protection group process input traffic, with the redundant spare(s) receiving periodic state updates" [54]. Therefore, from the architectural point of view, this tactic requires: (i) a router to ensure that only the active node process all the inputs, as well as to change the route to the redundant node(s) when there is a failure, and (ii) a periodic data controller in order for active and redundant node(s) to maintain periodic state updates. If there is a failure, the router selects a redundant spare after checking the state update. This tactic achieves a balance between the more highly available but more complex active redundancy tactic and the less available but significantly less complex spare tactic.

The PLA resulting is shown in Figure 11 and described as follows. The feature F1 is implemented by the component MeterCapturer, which reads text files of metering data associated to different energy resources, 


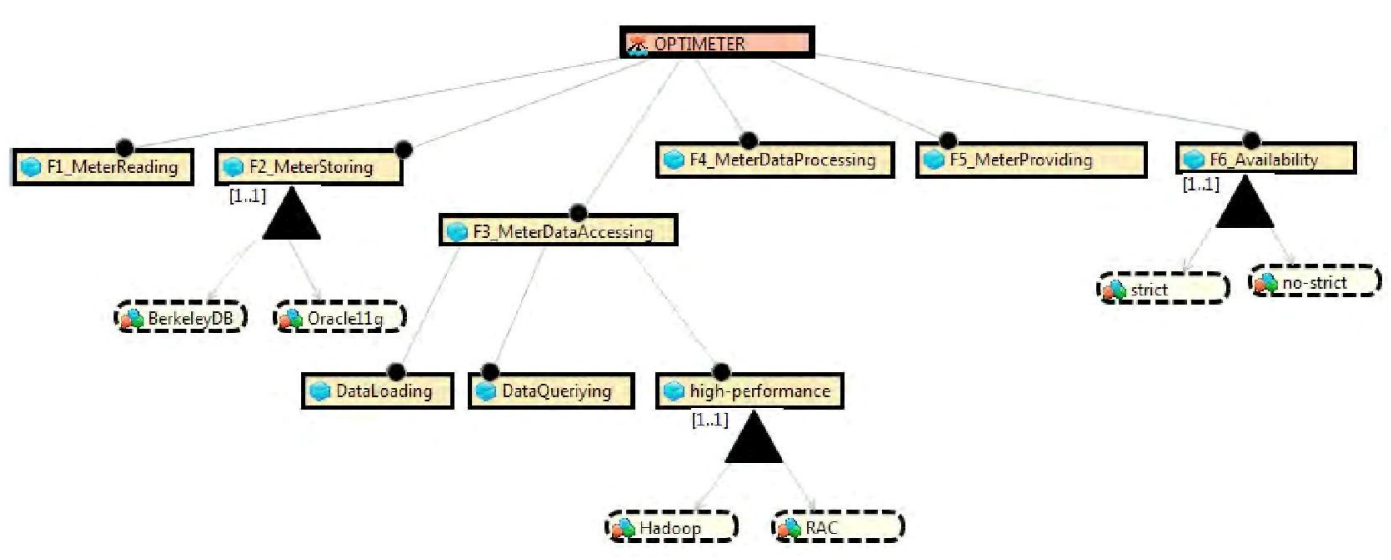

Fig. 10 Optimeter I - Feature model

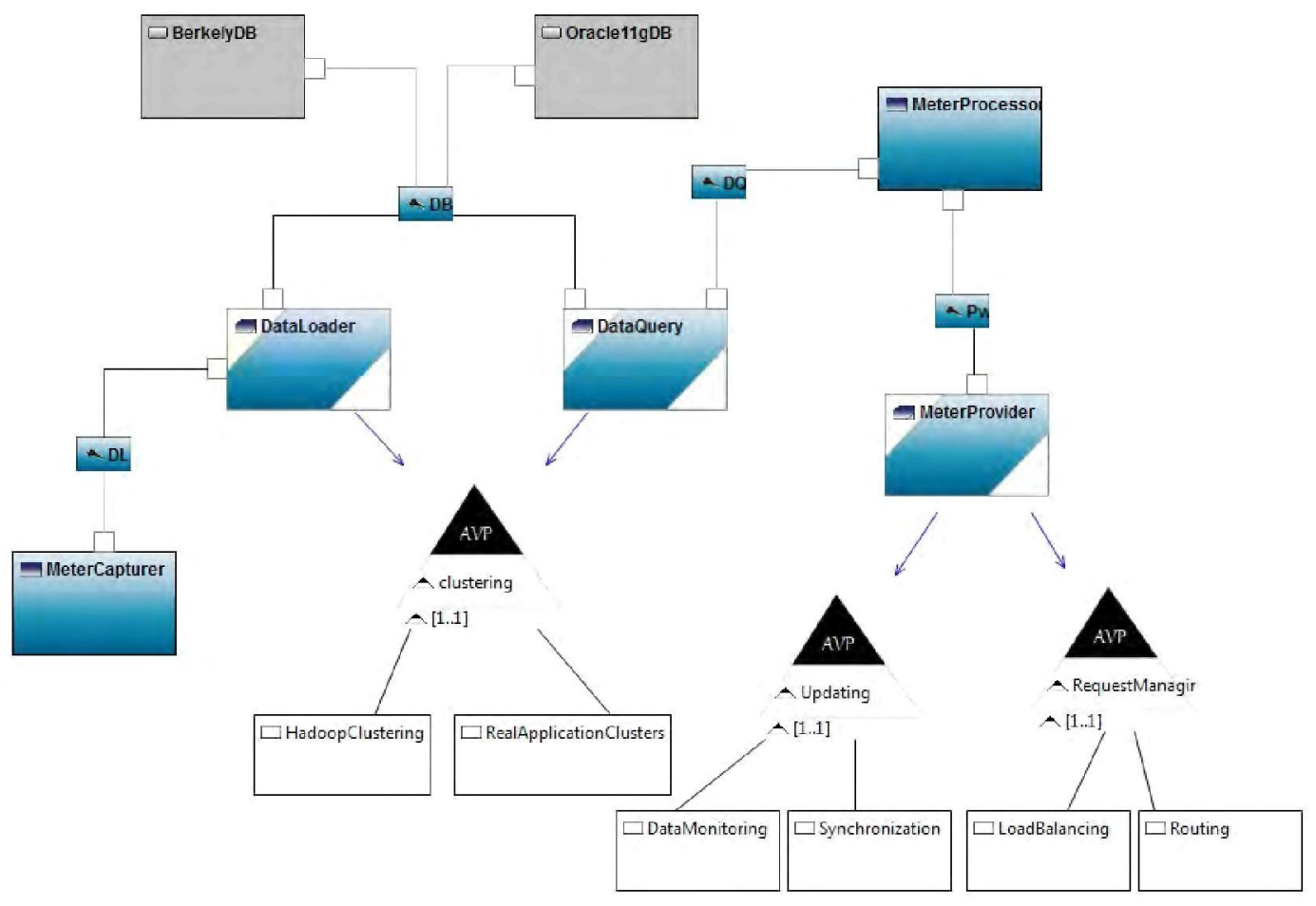

Fig. 11 Optimeter I - Flexible-PLA model

periods (quarterly, hourly, daily and monthly), and intervals, and processes the previously read data to form key/value pairs. The variability of the feature F2 is implemented by the optional components Berkeley $D B$ and Oracle $11 \mathrm{~g}$. The feature F3, and specifically the subfeatures DataLoading and DataQuerying are implemented by the PPCs DataLoader and DataQuery respectively. The architects took advantage of the PPC's variability mechanism to specify the variability of the feature high-performance as internal variability. This variability is internal to the PPCs DataLoader and DataQuery, i.e. the variability crosscut these two PPCs. Hence, both PPCs implement the variability of performance through the variability point clustering and the variants Hadoop-
Clustering and RealApplicationClusters (see Figure 11). These variants implement the operations for clustering and distributing work around a cluster to improve the data accessing performance (for loading and querying). Figure 13 shows an extract of the PPC DataLoader code and how internal variability works. Specifically, the figure shows how the code of the variant HadoopClustering is linked to the code of the PPC DataLoader through the weavings. The feature F4 is implemented by the component MeterProcessor, which implements the algorithms for validating metering data and calculating optimal vectors. Finally, the feature F5 is implemented by the PPC MeterProvider. Again, the architects took advantage of the PPC's variability mecha- 
nism to specify the variability of the feature $\mathrm{F} 6$ as internal variability to the PPC MeterProvider. This PPC implements the variability of availability through the variability points Updating and RequestManaging, and the variants DataMonitoring, Synchronization, LoadBalancing, and Routing.

The third activity of the case study execution was the definition of traceability links between the Optimeter feature model (see Figure 10) and the Optimeter Flexible-PLA model (see Figure 11). The resulting Feature-PLA Traceability model is described as follows (see Figure 12): The links $I D \_001$ and ID_002 trace the grouped features Berkely $D B$ and Oracle $11 \mathrm{~g}$ to the optional component BerkeleyDB and Oracle $11 \mathrm{~g} D B$, respectively. The links $I D \_001$ and $I D \_002$ trace the features DataQueriying and DataLoading to the PPCs DataLoader and DataQuery, respectively. The feature group that implements the variability of performance is traced to the variability point clustering through the link ID_005. The links $I D \_006$ and ID_007 trace the grouped features Hadoop and $R A C$ to the variants HadoopClustering and RealApplicationClusters, respectively. The feature group that implements the variability of availability is traced to the variability points $U p$ dating and RequestManaging through the link ID_008. The link ID_009 traces the grouped feature strict to the variants Synchronization and LoadBalancing. The link ID_010 traces the grouped feature non-strict to the variants DataMonitoring and Routing. All these traceability links store semantic knowledge. To gain readability, Figure 12 only shows the attributes satisfacing, who, and when. The value of the attribute satisfacing from all of these links is strong positive. This means that the architectural elements - components, PPCs, variantsinvolved in the links fully satisfy the expected functionality of the features also involved in the links. Finally, the link ID_011 traces the variant Synchronization to the feature high-performance. This link shows the value medium negative for the attribute satisfacing, which means that the synchronization may negatively affect to the performance.

Once the features, the PLA, and the traceability links were described and modeled by the SPL engineers, the following activities were the implementation and the testing (see the fourth activity in subsection 3.3). The resulting source code (such as the code shown in Figure 13) is also linked to the components specified in the Feature-PLA Traceability model. All these activities comprise a typical domain engineering process in which the commonality and the variability of a SPL is defined and realized [46]. The result is a common structure - the OPTIMETER SPL platform - from which a set of derivative products - metering management sys- tem applications - can be efficiently developed and produced.

Next, Optimeter II started. Each one of the two product owners involved in the case study selected to implement two different products:

- A metering management system running over Berkeley DB and Hadoop, which has to be strictly available $24 / 7$ (product $A)$.

- A metering management system running over Oracle $11 \mathrm{~g}$ DB and RAC, which has to be available $24 / 7$ but it is possible to relax this restriction (product $B)$.

At this time, the product engineers configured specific products according to the products specifications that the owners expected to get (see the fifth activity in subsection 3.3). This means that the product engineers bound the variability. To do this, the product engineers examined the Feature-PLA Traceability model to ensure that the binding was correctly performed according to the products specifications. Hence, the product engineers, by means of the link ID_009 in Figure 12, checked that the configuration of the product $\mathrm{A}$ requires the binding of the variants Synchronization and LoadBalancing in order to meet strict availability. They also checked, by means of the link ID_011 in Figure 12 that the variant Synchronization could affect the required high performance. Finally, the product engineers checked, by means of the link ID_010 in Figure 12, that the configuration of the product $B$ should bind the variants DataMonitoring and Routing which implement a variation less available but that does not jeopardize performance.

After selecting the specific variants for the products $\mathrm{A}$ and $\mathrm{B}$, the last activity (see the sixth activity in subsection 3.3) was performed as follows. This activity consisted of the generation of the code for the products $\mathrm{A}$ and $\mathrm{B}$, i.e. the binding of the variability at the code-level. To do this, the product engineers used the FPLA modeling framework to automatically generate the code for each one of these two products. Hence, for the product $A$ the weavings that insert the code of the variant Hadoop Clustering into the PPC DataLoader were automatically generated (see Figure 13). Similarly, for the product $B$ the weavings that insert the code of the variant RealApplicationClusters into the PPC DataLoader were automatically generated. In this way, the PPC DataLoader can be easily configured to support Hadoop clustering as shown in Figure 13, or to support Real Application Clusters.

The development of these two projects provided the necessary data to conduct the case study analysis and interpretation. 


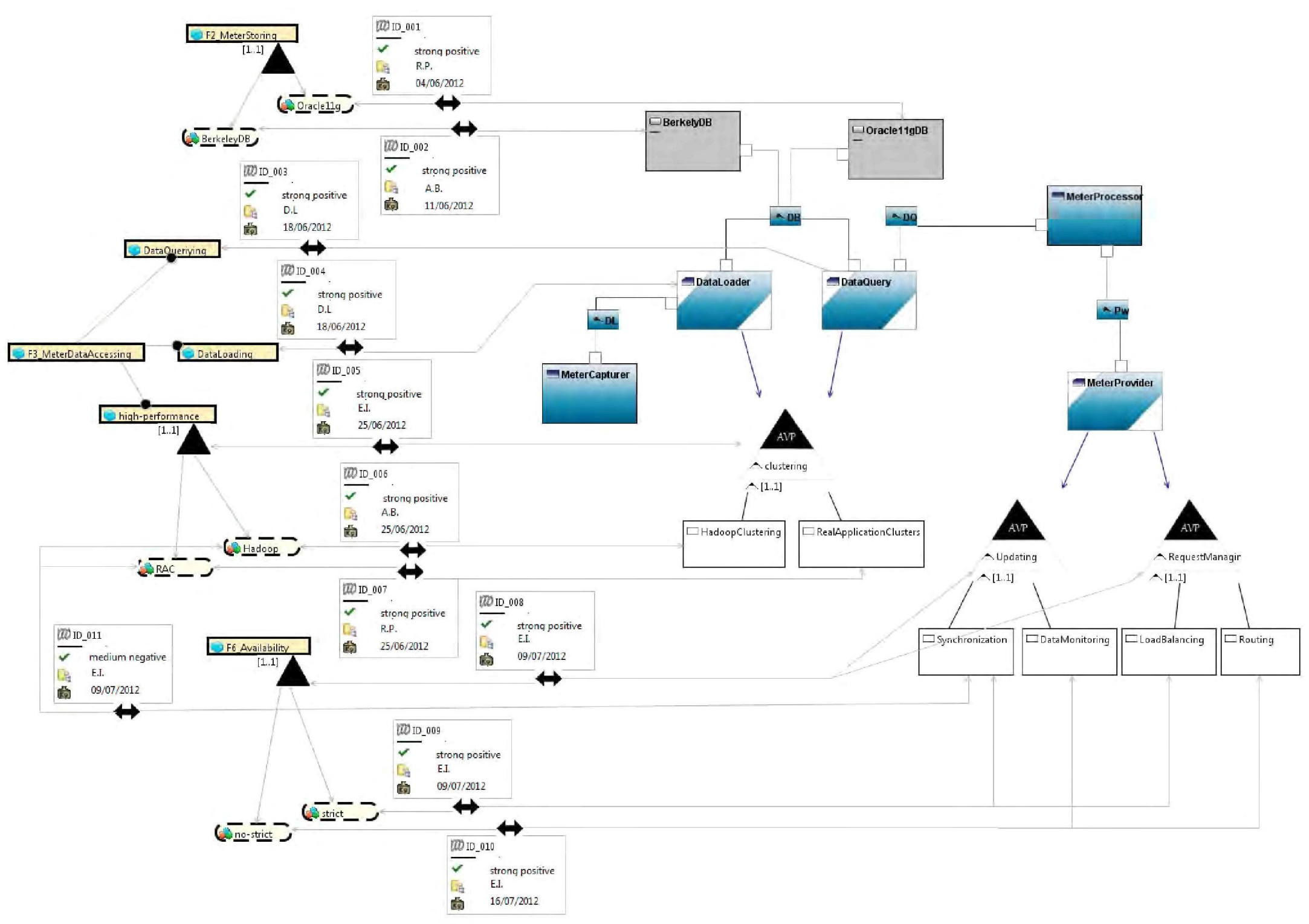

Fig. 12 Optimeter I - Feature-PLA Traceability model 


\subsubsection{Analysis and interpretation}

Quantitative and qualitative analysis was used to examine the data gathered during the case study. The data collected consisted of the models resulting from the projects (see Figures 10-12), archival data from Redmine, as well as the questionnaires and interviews performed with the SPL and product engineers. The analysis of these data has permitted to find evidence to answer each one of the research questions:

$R Q_{1}$ : Are Feature-PLA Traceability modeling primitives effective in providing SPL engineers the means for specifying traceability for most common kinds of variations that they define on their product family?

The evidence to answer $R Q_{1}$ is explored through descriptive statistics that measures the number of variations of interest for the SPL engineers that they were able to trace by using the modeling primitives provided by the Feature-PLA Traceability Model. The number of points of variability is three data storage, clustering, and availability - with a total of six variants - BerkeleyDB and Oracle11g for data storing, Hadoop and RAC for clustering, and finally strict and non-strict availability.

The traceability of the variability for data storing was well-supported through links between grouped features and optional components (see the links ID_001 and ID_002 in Figure 12). As the architects took advantage of the PPC's variability mechanism to specify internal variability of components - specifically to specify the variability of clustering and availability-, they required the capability of tracing this variability which is internal to one or more components. Hence, the SPL engineers were able to trace the variants Hadoop and RAC to the architectural elements that implement these two different clustering technologies through links between grouped features and variants (see the links ID_006 and ID_007 in Figure 12). The SPL engineers were also able to trace the variants strict and non-strict availability to the architectural elements that implement two different availability tactics with different repair timeactive and passive redundancy - through links between grouped features and variants (see the links ID_006 and ID_007 in Figure 12).

Therefore, as it can be verified in Figure 12, the SPL engineers were able to effectively trace all kinds of variations they required.

$R Q_{2}$ : Do Feature-PLA Traceability models assist and guide SPL engineer at the time of configuring the products of their product family?

The evidence to answer $R Q_{2}$ is assessed by analyzing the interviews given to the SPL and product engi- neers. From these interviews, the following excerpts can be highlighted:

$<<$ It could have been very difficult for us - the product engineers - to be able to determine a valid configuration for a metering management system application requiring strict or non-strict availability without the use of the Feature-PLA Traceability model (see Figure 12$)>>$.

This means that the use of the Feature-PLA Traceability model of Figure 12 was particularly useful for the product engineers to understand the system as they hadn't been developed the OPTIMETER SPL platform.

$<<$ To configure the products $\mathrm{A}$ and $\mathrm{B}$ we needed knowledge that helped us to perform the binding according to their respective requirements. Without the knowledge provided by the Feature-PLA Traceability model (see Figure 12), it may had been difficult to know (i) if a metering management system application requiring strict availability had to implement the services for synchronization and load balancing, or (ii) if a metering management system application requiring non-strict availability had to implement the services for routing and data monitoring. This means, without the traceability model, we hadn't feel confident about whether the variants we bound implemented all the services to satisfy the requirements of the products $\mathrm{A}$ and B. So, the traces between (i) the feature strict availability to the variants Synchronization and LoadBalancing, and (ii) the feature non-strict availability to the variants Routing and DataMonitoring, were really useful to ensure that the ginding of variability was realized correctly $>>$.

$<<$ Feature-PLA Traceability models may be useful to identify where a feature is implemented in the PLA. As a result, it may also be useful to identify, given a change in a feature, where the change impacts the PLA. From the Feature-PLA Traceability model of Figure 12 it is easy to observe that a change in the tactic to implement strict availability may impact the variants Synchronization and LoadBalancing. Perhaps this is not easy to locate in the code, but by making it available at the architecture-level, Feature-PLA Traceability models facilitate this task. This impact knowledge may help us to correctly implement a change while maintaining the integrity of the architecture $>>$.

These excerpts from the SPL and product engineers put in evidence that our solution for tracing variability assisted and helped them at the time of configuring the two metering management systems (products A and B) from the OPTIMETER PLA. 


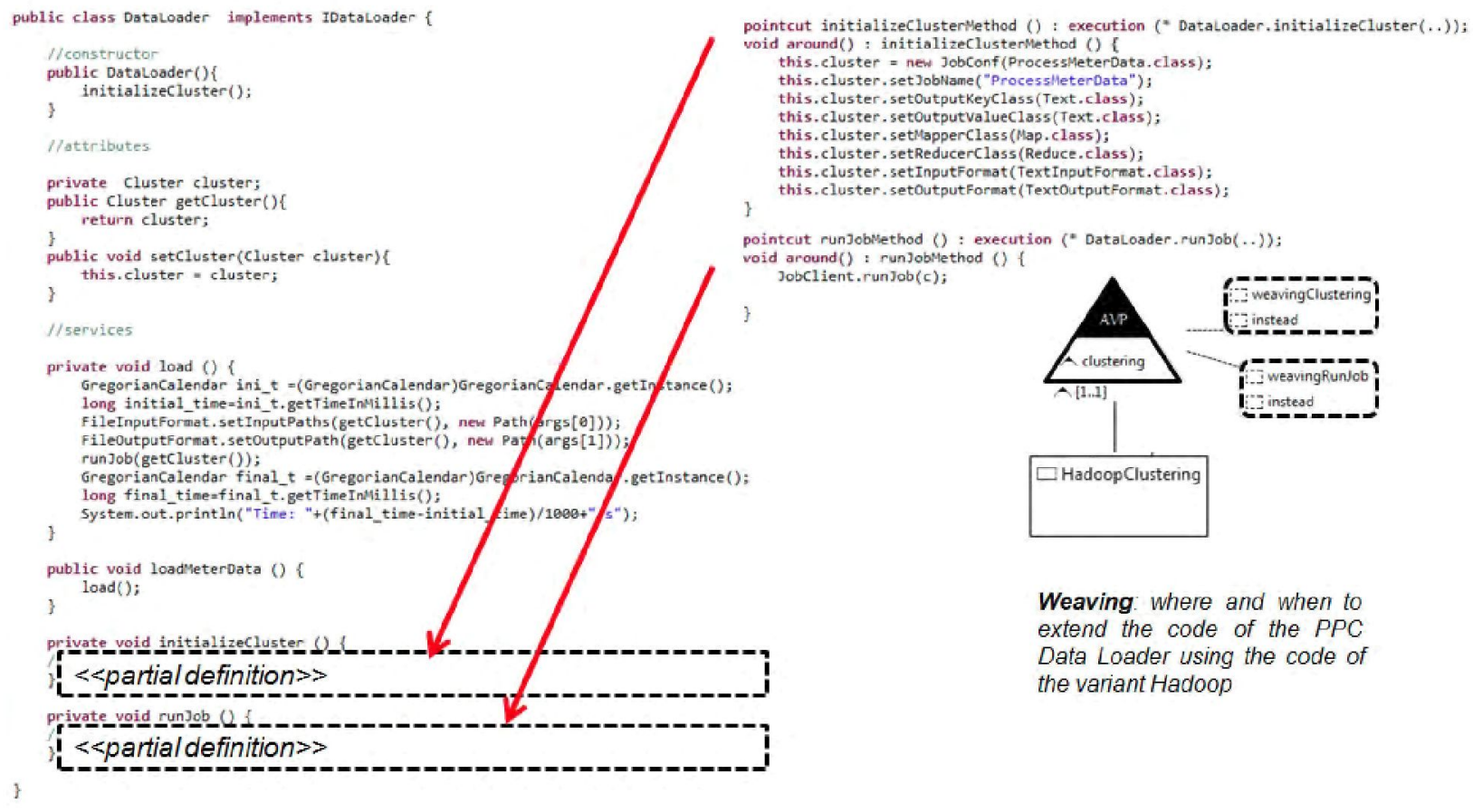

Fig. 13 Optimeter I - PPC DataLoader

\subsubsection{Evaluation of validity}

Case studies are qualitative in nature. For this reason, collected data from case studies are usually very difficult to be objectively judged [61]. To improve the internal validity of the results presented, the independent variables that could influence this case study have been identified as follows: The engineer's experience has a great influence. Its influence has been reduced as the expertise of the engineers who participated in the case study were very different (1 year vs. 7 years). However, the influence of project's size and architecture's complexity cannot be reduced due to the inherent nature of case studies, which normally focus on one project. Also to improve the internal validity of the results, triangulation of source data has been used to increase the reliability of the results. In this regard, interviews were individually conducted with the engineers, although several questions were asked in a group setting to encourage discussion.

Construct validity is concerned with the procedure to collect data and with obtaining the right measures for the concept being studies. It addresses among others misinterpretation of interview questions which was mitigated by discussing the interpretations of interviews with the interviewees to validate them.

However, the major limitation in case study research concerns external validity, i.e. "the generality of the results with respect to a specific population" [22], as only one case is studied. In return, case studies allow one to evaluate a phenomenon, a model, or a process in a real setting. This is something important in software engineering in which a multitude of external factor may affect to the validation results, and that other techniques such as formal experiments, although they permit replication and generalization, do not consider as they are conducting under controlled settings.

Reliability is concerned with replication, in case studies with the fact that the same results would be found if re-doing the analysis. This is why interviews were recorded and interpretations were reviewed by other participants in the study in order to avoid researcher bias.

\subsection{Case Study Conclusions}

We obtained evidence of the viability of the FeaturePLA Traceability Model through the execution of a case study performed in an experimental laboratory called i-Smart Software Factory. It combines both academic and industrial efforts in $\mathrm{R} \& D$, with remarkable facilities for tracking the projects' progress. The case study puts the proposed traceability solution into practice within the development of a SPL of power metering management systems for Smart Grids. The results show evidence of that (i) the Feature-PLA Traceability modeling primitives were effective in providing the capabilities for tracing most common kinds of variations that the SPL engineers required define, and (ii) the Feature- 
PLA Traceability provided knowledge that helped the product engineers to make better decisions at the time of configuring the products A and B during Optimeter II as they did not know the OPTIMETER SPL platform because they had not participated on its construction during Optimeter I. These promising results did not interfere with other practices and did not incur a big cost, making traceability possible. However, the use of the Feature-PLA Traceability Model requires to know and understand the modeling concepts on which they are based on, as well as to learn the usage of the FPLA modeling framework. The learning curve of these concepts as well as the usage of FPLA could slow down the process of putting traceability into practice. In fact, the SPL engineers expressed reluctance at the time of putting traceability into practice, although later, the product engineers found this traceability essential to do their work during the configuration of variability to derive the products $\mathrm{A}$ and $\mathrm{B}$.

\section{Related Work}

Recently, there has been a growing recognition of the importance of traceability in SPLE, which has resulted in more and more research in this area. Hence, Moon et al. [38] defined a Variability Trace Metamodel that connects two metamodels: a metamodel for requirements and a metamodel for architecture. Ajila et al. [3] presented an evolution model that defines a dependency relationship structure of various SPL artifacts. Satyananda et al. [52] presented a framework for formally identifying traceability between feature and architecture models using Formal Concept Analysis, functional decomposition, and a set of mapping analysis rules. Finally, Berg et al. [10] also defined a conceptual variability model that captures variability information across the various artifacts involved in the SPLE development. All these approaches ${ }^{16}$ offer support for tracing SPL, including traceability of variability. The granularity of traceability links relies largely on the granularity of elements to be traced, whether requirements, architectural elements, or classes. The approaches before mentioned support architectural variability by adding or removing components or connections. However, these approaches do not have the capabilities for tracing the variability that is internal to components, i.e. variations that have fine granularity and cannot be designed as components. In this sense, our traceability model takes an step forward due to the fact that it is based on the FlexiblePLA Model which allows SPL engineers to specify both

\footnotetext{
16 Although other papers propose other traceability approaches $[49,47,42]$, we did not include them here as they do not consider SPLE.
}

external and internal variability thanks to the PPC's variability mechanism. The fact that internal variability can crosscut several components, and that is modularized and reused by PPCs (i.e. this variability is not scattered through these components), makes it easier its traceability. Therefore, our approach makes both coarse-grained and fine-grained traceability possible.

Additionally, Satyananda et al. [52] defined a set of mapping analysis rules similar to the linkage rules we propose. These rules are textually described while the linkage rules we propose are formally stated by the Feature-PLA Traceability Model. Models are completely subject to automation, which (i) makes it easier to define traceability links while their correctness is guaranteed by model-conformation, (ii) promotes learning and reasoning over the knowledge they contain, and (iii) provides the capabilities to (semi-)automatically generate other artifacts, such as code, through model transformations.

Finally, it is important to mention the work of Anquetil et al. [4] that defined a common traceability framework across the various activities of SPL development and specified a metamodel for a repository of traceability links. This framework provides a big picture of traceability for SPL by offering modeling primitives for tracing any artifact involved in the SPL development. This complete framework does not embed all these artifacts but embed references to them in order to make manageable the high number of artifacts that a complete SPL construction requires to trace. As a result, sources and targets of traceability links are paths where the artifacts are stored or can be found (documents, diagrams or classes). The fact that these artifacts are external to the traceability model makes it difficult to guarantee that a change in an artifact is also updated in the traceability model. Additionally, this artifacts outsourcing makes it difficult to understand the traceability models and their usage as a guidance during the configuration of the products of a SPL while ensuring that the variability binding meets the product requirements. This is due to the fact that the relationships inside artifacts (e.g. a feature has a XOR feature group) are not included in the traceability framework and traceability links do not contain rationale and information about the traceability-making process. The Flexible-PLA Traceability Model reduces its scope by focusing on the traceability between feature and PLA models and prioritizes the knowledge and guidance that traceability models can provide during SPL product configuration to ensure the requirements compliance. This is supported by including the source and target artifacts - the Feature and PLA models - into the traceability model, as well as their relationships, and en- 
riching traceability links with rationale and information about the traceability-making process.

\section{Conclusions and Further Work}

SPLE is facing new challenges, being one of the most important the traceability of variability. To deal with this challenge, this paper presents a solution for tracing Feature and PLA models called Feature-PLA Traceability Model, as well as the modeling framework that support it. The Feature-PLA Traceability Model defines a set of linkage rules to trace variable features to both the coarse-grained variability of complex componentsexternal variability - and the fine-grained variability of simple components - internal variability.

The description and the traceability of the variability that is internal to one or many components is as important as the description and the traceability of the external variability. It is essential to cope with most kinds of variation that SPL engineers could define on their product families. Supporting both coarse-grained and fine-grained traceability of variability helps product engineers at the time of configuring this variability to derive products. This means that product engineers can examine Feature-PLA Traceability models to ensure that variability bindings satisfy the product requirements.

As future work, the knowledge stored in FeaturePLA Traceability models could be used to analyze the impact of changing requirements, i.e. to analyze how a change in features may affect the architecture by traversing the traces that link them. this was suggested by the engineers involved in the case study during the interviews. Additionally, the knowledge currently stored could be extended to capture more types of knowledge, such as domain knowledge, design decisions, assumptions, etc.

The Feature-PLA Traceability model and its usage still have several limitations that should be addressed in the near future. The main one is scalability, such as a scalable visualization. However this limitation is more related to the algorithms to leverage and visualize the traceability knowledge than the expressiveness of the traceability model.

Acknowledgements The work reported in here has been partially sponsored by the Spanish fund: INNOSEP (TIN200913849), IMPONET (ITEA 2 09030, TSI-02400-2010-103), iSSF (IPT-430000-2010-038), NEMO\&CODED (ITEA2 08022, IDI-20110864) and ENERGOS (CEN-20091048). Finally, it is also funded by the UPM (Technical University of Madrid) under their Researcher Training program.

\section{References}

1. Adachi Barbosa, E., Batista, T., Garcia, A., Silva, E.: Plaspectualacme: An aspect-oriented architectural description language for software product lines. In: I. Crnkovic, V. Gruhn, M. Book (eds.) Software Architecture, Lecture Notes in Computer Science, vol. 6903, pp. 139-146. Springer Berlin / Heidelberg (2011)

2. Aizenbud-Reshef, N., Nolan, B.T., Rubin, J., ShahamGafni, Y.: Model traceability. IBM Systems Journal 45(3), $515-526$ (2006). DOI 10.1147/sj.453.0515

3. Ajila, S., Kaba, A.: Using traceability mechanisms to support software product line evolution. In: Information Reuse and Integration, 2004. IRI 2004. Proceedings of the 2004 IEEE International Conference on, pp. 157-162 (2004). DOI 10.1109/IRI.2004.1431453

4. Anquetil, N., Kulesza, U., Mitschke, R., Moreira, A., Royer, J.C., Rummler, A., Sousa, A.: A modeldriven traceability framework for software product lines. Software and Systems Modeling p. 25 (June 2009). DOI 10.1007/s10270-009-0120-9. URL http://www.springerlink.com/content/wvm4hv8r78117785

5. Antkiewicz, M., Czarnecki, K.: Featureplugin: feature modeling plug-in for eclipse. In: eclipse '04: Proceedings of the 2004 OOPSLA workshop on eclipse technology eXchange, pp. 67-72. ACM, New York, NY, USA (2004). DOI $10.1145 / 1066129.1066143$

6. Bachmann, F., Bass, L.: Managing variability in software architectures. In: SSR '01: Proceedings of the 2001 symposium on Software reusability, pp. 126-132. ACM, New York, NY, USA (2001). DOI 10.1145/375212.375274

7. Bachmann, F., Goedicke, M., Leite, J., Nord, R., Pohl, K., Ramesh, B., Vilbig, A.: A meta-model for representing variability in product family development. In: F. van der Linden (ed.) Software Product-Family Engineering, Lecture Notes in Computer Science, vol. 3014, pp. 66-80. Springer Berlin / Heidelberg (2004)

8. Bass, L., Clements, P., Kazman, R.: Software Architecture in Practice, 2nd edition. Addison-Wesley Pearson Education (2003)

9. Benavides, D., Segura, S., Ruiz-Cortés, A.: Automated analysis of feature models 20 years later: A literature review. Inf. Syst. 35(6), 615-636 (2010)

10. Berg, K., Bishop, J., Muthig, D.: Tracing software product line variability: from problem to solution space. In: SAICSIT '05: Proceedings of the 2005 annual research conference of the South African institute of computer scientists and information technologists on IT research in developing countries, pp. 182-191. South African Institute for Computer Scientists and Information Technologists, , Republic of South Africa (2005)

11. Beydeda, S., Book, M., Gruhn, V.: Model-Driven Software Development. Springer (2005)

12. Bezivin, J.: On the unification power of models. Software and Systems Modeling 4(2), 171-188 (2005)

13. Cleland-Huang, J., Gotel, O., Zisman, A. (eds.): The Grand Challenge of Traceability (v1.0). Springer-Verlag London Limited (2012)

14. Clements, P., Northrop, L.: Software Product Lines: Practices and Patterns. Addison-Wesley (2002)

15. Czarnecki, K.: Mapping features to models: A template approach based on superimposed variants. In: GPCE 2005 - Generative Programming and Component Enginering. 4th International Conference, pp. 422-437. Springer (2005)

16. Dashofy, E.M., Hoek, A.v.d.: Representing product family architectures in an extensible architecture description 
language. In: PFE '01: Revised Papers from the 4th International Workshop on Software Product-Family Engineering, pp. 330-341. Springer-Verlag (2002)

17. Díaz, J., Pérez, J., Garbajosa, J., Yagüe, A.: Changeimpact driven agile architecting. In: Proceedings of the 46th Hawaii International Conference on System Sciences (HICSS '13), Hawaii, USA, January 7-10, 2013, pp. 47804789. IEEE Computer Society Press (2013)

18. Espinoza, A., Garbajosa, J.: A proposal for defining a set of basic items for project-specific traceability methodologies. In: Software Engineering Workshop, 2008. SEW '08. 32nd Annual IEEE, pp. 175-184 (2008)

19. Gotel, O., Finkelstein, C.: An analysis of the requirements traceability problem. In: Proceedings of the First International Conference on Requirements Engineering, pp. 94 -101 (1994). DOI 10.1109/ICRE.1994.292398

20. Gotel, Orlena et al.: The grand challenge of traceability (v1.0). In: J. Cleland-Huang, O. Gotel, A. Zisman (eds.) Software and Systems Traceability, pp. 343-409. Springer London (2012)

21. Hauser, J.R., Clausing, D.: The house of quality. Harvard Business Review 66(3), 63-73 (1988). Available through Harvard Business School Publishing.

22. van Heesch, U., Avgeriou, P., Hilliard, R.: A documentation framework for architecture decisions. Journal of Systems and Software 85(4), 795 - 820 (2012). DOI $10.1016 /$ j. jss. 2011.10 .017

23. van der Hoek, A., Heimbigner, D., Wolf, A.L.: Capturing architectural configurability: Variants, options, and evolution. Tech. rep., Technical Report CU-CS-895-99, Department of Computer Science, University of Colorado, Boulder, Colorado (1999)

24. Jacobson, I., Griss, M., Jonsson, P.: Software Reuse. Architecture, Process and Organization for Business Success. Addison-Wesley (1997)

25. Kang, K.C., Cohen, S.G., Hess, J.A., Novak, W.E., Peterson, A.S.: Feature-oriented domain analysis (foda) feasibility study. Tech. rep., Carnegie-Mellon University, Pittsburgh, PA, USA, CMU/SEI-90-TR-21 ESD-90-TR$222(1990)$

26. Khurum, M., Gorschek, T.: A systematic review of domain analysis solutions for product lines. J. Syst. Softw. 82(12), 1982-2003 (2009)

27. Kizcales, G., Lamping, J., Mendhekar, A., Maeda, C.: Aspect-oriented programming. In: Proceedings of the 11th European Conference on Object-Oriented Programming (ECOOP), Lecture Notes in Computer Science, vol. 1241. Springer-Verlag (1997)

28. Kolovos, D.S., Paige, R.F., Polack, F.A.C.: On-demand merging of traceability links with models. In: In Proc. of 3 rd ECMDA Traceability Workshop (2006)

29. Letelier, P.: A framework for requirements traceability in uml-based projects. In: In Proc. of 1st Intl. Workshop on Traceability in Emerging Forms of Softw. Eng, pp. 32-41 (2002)

30. Loughran, N., Sánchez, P., Garcia, A., Fuentes, L.: Language support for managing variability in architectural models. In: SC'08: Proceedings of Software Composition, 7th International Symposium, Lecture Notes in Computer Science, vol. 4954, pp. 36-51. Springer (2008)

31. Magee, J., Kramer, J.: Dynamic structure in software architectures. In: Proceedings of the 4th ACM SIGSOFT symposium on Foundations of software engineering, SIGSOFT '96, pp. 3-14. ACM, New York, NY, USA (1996)

32. Mahdavi-Hezavehi, S., Galster, M., Avgeriou, P.: Variability in quality attributes of service-based software systems: A systematic literature review. Information and Software Technology 55(2), 320-343 (2013)
33. Martin, J.L., Yague, A., Gonzalez, E., Garbajosa, J.: Making software factory truly global: the smart software factory project. In: F. Fagerholm (ed.) Software Factory Magazine. Available on http://www.softwarefactory.cc/magazine, p. 19 (2010)

34. Massoud Amin, S., Wollenberg, B.: Toward a smart grid: power delivery for the 21st century. Power and Energy Magazine, IEEE 3(5), 34-41 (2005). DOI 10.1109/MPAE.2005.1507024

35. Matinlassi, M.: Comparison of software product line architecture design methods: Copa, fast, form, kobra and qada. In: ICSE '04: Proceedings of the 26th International Conference on Software Engineering, pp. 127-136. IEEE Computer Society, Washington, DC, USA (2004)

36. Medvidovic, N., Taylor, R.N.: A classification and comparison framework for software architecture description languages. IEEE Trans. Softw. Eng. 26(1), 70-93 (2000). DOI http://dx.doi.org/10.1109/32.825767

37. Mens, T.: Future Research Challenges in Software Evolution and Maintenance - Report from EC Expert Meeting. ERCIM News 81 (2010)

38. Moon, M., Chae, H.S., Nam, T., Yeom, K.: A metamodeling approach to tracing variability between requirements and architecture in software product lines. In: CIT '07: Proceedings of the 7th IEEE International Conference on Computer and Information Technology, pp. 927-933. IEEE Computer Society, Washington, DC, USA (2007)

39. Object Management Group: Data distribution service for real-time systems, v1.2 (2006)

40. Object Management Group: Meta-Object Facility (MOF) Specification 2.0 TR formal-06-01-01. http://www.omg.org/spec/MOF/2.0/PDF/ (2006)

41. Object Management Group: OCL Specification Version 2.2. http://www.omg.org/spec/OCL/2.2/ (2011)

42. Olsen, G., Oldevik, J.: Scenarios of traceability in model to text transformations. In: D. Akehurst, R. Vogel, R. Paige (eds.) Model Driven Architecture- Foundations and Applications, Lecture Notes in Computer Science, vol. 4530, pp. 144-156. Springer Berlin / Heidelberg (2007)

43. van Ommering, R., van der Linden, F., Kramer, J., Magee, J.: The koala component model for consumer electronics software. Computer 33(3), 78-85 (2000). DOI $10.1109 / 2.825699$

44. Pérez, J., Díaz, J., Garbajosa, J., Alarcón, P.P.: Flexible working architectures: Agile architecting using ppcs. In: Proceedings of the 4th European Conference on Software Architecture (ECSA 2010), LNCS, pp. 102-117. Springer-Verlag, Berlin, Heidelberg (2010)

45. Pérez, J., Díaz, J., Soria, C.C., Garbajosa, J.: Plastic partial components: A solution to support variability in architectural components. In: Proceedings of Joint Working IEEE/IFIP Conference on Software Architecture 2009 and European Conference on Software Architecture 2009 WICSA/ECSA 2009, Cambridge, UK, 14-17 September 2009, pp. 221-230. IEEE (2009)

46. Pohl, K., Böckle, G., Linden, F.: Software Product Line Engineering: Foundations, Principles and Techniques. Springer, Germany (2005)

47. Pohl, K., Brandenburg, M., Gülich, A.: Integrating requirement and architecture information: A scenario and meta-model approach. In: REFSQ'01: Proceedings of The Seventh International Workshop on Requirements Engineering: Foundation for Software Quality, pp. 68-84 (2001)

48. Poshyvanyk, D., Di Penta, M., Kagdi, H.: Sixth international workshop on traceability in emerging forms of 
software engineering: (tefse 2011). In: 33rd International Conference on Software Engineering (ICSE 2011), pp. $1214-1215$ (2011). DOI 10.1145/1985793.1986052

49. Ramesh, B., Jarke, M.: Toward reference models for requirements traceability. IEEE Trans. Softw. Eng. 27(1), 58-93 (2001). DOI http://dx.doi.org/10.1109/32.895989

50. Runeson, P., Höst, M.: Guidelines for conducting and reporting case study research in software engineering. Empirical Software Engineering 14, 131-164 (2009)

51. Runeson, P., Höst, M., Rainer, A., Regnell, B.: Case Study Research in Software Engineering: Guidelines and Examples. John Wiley \& Sons (2012)

52. Satyananda, T.K., Lee, D., Kang, S., Hashmi, S.I.: Identifying traceability between feature model and software architecture in software product line using formal concept analysis. In: Proceedings of the International Conference Computational Science and its Applications, pp. 380 388. IEEE Computer Society, Washington, DC, USA (2007)

53. Schwaber, K., Beedle, M.: Agile Software Development with Scrum. Prentice-Hall (2002)

54. Scott, J., Kazman, R.: Realizing and refining architectural tactics: Availability. Tech. rep., CMU/SEI-2009TR-006 ESC-TR-2009-006 (2009)

55. Selic, B.: The pragmatics of model-driven development. IEEE Softw. 20(5), 19-25 (2003). DOI http://dx.doi.org/10.1109/MS.2003.1231146

56. Staab, S., Walter, T., Grner, G., Parreiras, F.: Model driven engineering with ontology technologies. In: U. Amann, A. Bartho, C. Wende (eds.) Reasoning Web. Semantic Technologies for Software Engineering, Lecture Notes in Computer Science, vol. 6325, pp. 62-98. Springer Berlin Heidelberg (2010)

57. Stake, R.E.: The Art of Case Study Research. SAGE Publications Inc (1995)

58. Szyperski, C.: Component Software: Beyond ObjectOriented Programming. Addison-Wesley Longman Publishing Co., Inc., Boston, MA, USA (2002)

59. Taha, W.M.: Domain-Specific Languages IFIP TC 2 Working Conference, DSL, Lecture Notes in Computer Science, vol. 5658. Springer Berlin / Heidelberg (2009)

60. Weiler, T.: Modelling architectural variability for software product lines. In: SVM'03: Proceedings of the Software Variability Management Workshop, pp. 53-61 (2003)

61. Yin, R.: Case study research. Design and methods. 4th ed. London, Sage (2008) 